Discrete Comput Geom 38:355-387 (2007)

DOI: $10.1007 / \mathrm{s} 00454-007-1342-7$

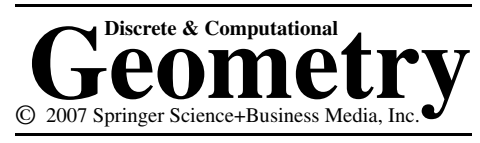

\title{
Four-Dimensional Regular Polyhedra
}

\author{
Peter McMullen \\ Department of Mathematics, University College London, \\ Gower Street, London WC1E 6BT, England \\ p.mcmullen@ucl.ac.uk
}

\begin{abstract}
This paper completes the classification of the four-dimensional (finite) regular polyhedra, of which those with planar faces were-in effect-found by Arocha, Bracho and Montejano. However, the methods employed here are in the same spirit as those used in the description of all three-dimensional regular polytopes by this author and Schulte, and the regular polytopes of full rank by this author. The procedure has two stages. First, the possible dimension vectors $\left(\operatorname{dim} R_{0}, \operatorname{dim} R_{1}, \operatorname{dim} R_{2}\right.$ ) of the mirrors $R_{0}, R_{1}, R_{2}$ of the generating reflexions of the symmetry groups are determined. Second, all polyhedra with a given dimension vector are found. Most of the polyhedra are related to four-dimensional Coxeter groups, although one class has to be approached using quaternions.
\end{abstract}

\section{Introduction}

Regular polyhedra have been a subject of fascination since classical times. Apart from their intrinsic interest as highly symmetric objects (see, for example, [4]), the so-called Platonic solids play an important rôle in areas such as isoperimetry (see [11]). In this paper it is the former aspect, suitably generalized, which concerns us.

In [21] (see also Sections 7E and 7F of [22]), the present author and Schulte classified completely all the faithfully realized regular polytopes and discrete regular apeirotopes in dimensions up to three. Further, in [18], this author classified the regular polytopes and apeirotopes of full (that is, maximal) rank in each higher dimension. This paper continues that line of investigation, by finding all the four-dimensional (finite) regular polyhedra.

The context of the paper is that of realized regular polytopes, the general background of which is described in Chapter 5 of [22]. There are two quite different ways to approach realizations. The first, for which a fairly complete theory exists (at least, in the finite case), asks for a description of the space of all realizations of a given abstract regular polytope or apeirotope, with rank playing only a minor rôle (see Sections 5B and 5C of [22] for further details). The second, about which much less is known in general terms, asks for 
a classification of the realizations of all these polytopes and apeirotopes in a euclidean space of given dimension (in this case, it is usual to impose conditions such as faithfulness and discreteness).

The latter problem is solved in up to three dimensions. The finite regular polyhedra have long been known; adding to the Petrie-Coxeter apeirohedra of [3], Grünbaum [13] found all but one of the remaining regular apeirohedra, while Dress [8], [9] found the missing example, and proved that the classification was then complete. (For polyhedra which are "regular" in a more general sense, see [14].) We refer the reader to [21] for a quick method of arriving at the full characterization, including a discussion of the geometry of the regular apeirohedra and presentations of their symmetry groups, as well as for the enumeration of the regular 4-apeirotopes in three dimensions. In addition, the regular polytopes and apeirotopes of full (that is, maximal) rank in each dimension were classified in [18], using a refinement of earlier techniques.

In four dimensions, the currently open problems are those of classifying the finite regular polyhedra, and the regular apeirohedra and 4-apeirotopes; [18] solves the problems of the regular 4-polytopes and 5-apeirotopes. The present paper settles the first of these problems (the polyhedra with planar faces were-in effect—classified in [1] and [2]), again using similar techniques. The core of the argument consists of determining the possible sequences $\left(\operatorname{dim} R_{0}, \operatorname{dim} R_{1}, \operatorname{dim} R_{2}\right)$ - the dimension vectors-of the mirrors of the reflexions $R_{0}, R_{1}, R_{2}$ which generate the symmetry groups of the regular polyhedra. The rest consists of finding each polyhedron with a given dimension vector.

A brief outline of the contents of this paper appeared in Section 7 of [23]; mistakes and omissions made there have been corrected here.

The problem of classifying four-dimensional chirol polyhedra (which have only "rotational" symmetry) is also open; for their three-dimensional (infinite) analogues, see [25] and [26].

\section{Regular Polytopes}

For the general background on abstract regular polytopes, we refer the reader to the monograph [22]; for the most part, we shall not cite original papers on the theory of abstract regular polytopes directly. In this paper, we largely concentrate on the geometric aspects of the theory, that is, on realizations of regular polytopes.

There are many candidates for spaces in which regular polytopes $\mathcal{P}$ might be realized geometrically. The usual (and generally most useful) context of realizations is of those in euclidean spaces, because it is in these that we obtain the richest structure. (Note, however, that some basic results have a common statement if they are posed for realizations in spherical or hyperbolic spaces as well; see Section 3 of [18].) In fact, since we consider realizations in $\mathbb{E}^{4}$ of (finite) regular polyhedra (3-polytopes) alone here, we later tailor our description accordingly.

It is a familiar fact (see Theorem 2E11 of [22]) that an abstract regular $n$-polytope $\mathcal{P}$ can be identified with its automorphism group, which is a string $C$-group $\Gamma=$ $\left\langle\rho_{0}, \ldots, \rho_{n-1}\right\rangle$ generated by $n$ involutions $\rho_{j}$ (the distinguished generators), such that $\rho_{j}$ and $\rho_{k}$ commute if $0 \leqslant j \leqslant k-2 \leqslant n-3$, and

$$
\left\langle\rho_{i} \mid i \in \mathrm{J}\right\rangle \cap\left\langle\rho_{i} \mid i \in \mathbf{K}\right\rangle=\left\langle\rho_{i} \mid i \in \mathrm{J} \cap \mathbf{K}\right\rangle
$$


for any $\mathrm{J}, \mathrm{K} \subseteq \mathrm{N}:=\{0, \ldots, n-1\}$; the last is the intersection property. The $j$-faces of $\mathcal{P}$ are then the right cosets $\Gamma_{j} \sigma$ of the distinguished subgroup

$$
\Gamma_{j}:=\left\langle\rho_{i} \mid i \neq j\right\rangle
$$

for each $j \in \mathrm{N}$, and two faces are incident just when they intersect (as cosets). In fact, incidence actually induces an order relation:

$$
\Gamma_{j} \sigma \leqslant \Gamma_{k} \tau \quad \Leftrightarrow \quad \Gamma_{j} \sigma \cap \Gamma_{k} \tau \neq \emptyset \quad \text { and } \quad j \leqslant k .
$$

Formally, we also adjoin two copies of $\Gamma$ itself, labelled $\Gamma_{-1}$ and $\Gamma_{n}$, as the (unique) $(-1)$ - and $n$-faces of $\mathcal{P}$.

The maximal chains (with respect to this ordering) are the flags of $\mathcal{P}$; the group $\Gamma$ is then simply transitive on the flags of $\mathcal{P}$. (Note that we do not usually mention the $(-1)$ and $n$-faces in flags.) With appropriate conditions on the poset of faces of an abstract polytope $\mathcal{P}$ (see Section 2A of [22]), this latter condition can be used as a definition of regularity of $\mathcal{P}$. Calling two flags adjacent if they differ by one face, the distinguished generator $\rho_{j}$ takes the base flag $\Phi:=\left\{\Gamma_{0}, \ldots, \Gamma_{n-1}\right\}$ into the flag $\Phi^{j}$ which differs from it in $\Gamma_{j}$.

Note that the distinguished subgroups $\Gamma_{n-1}=\left\langle\rho_{0}, \ldots, \rho_{n-2}\right\rangle$ and $\Gamma_{0}=$ $\left\langle\rho_{1}, \ldots, \rho_{n-1}\right\rangle$ are themselves string C-groups; the corresponding polytopes are the facet and vertex-figure of $\mathcal{P}$, respectively.

To avoid cases which, in our context, turn out to be trivial, we usually assume that adjacent generators $\rho_{j-1}$ and $\rho_{j}$ of $\Gamma$ do not commute (this is justified almost immediately). In other words, the period $p_{j}$ of the product $\rho_{j-1} \rho_{j}$ satisfies $p_{j}>2$ (our polytopes are finite, and so $p_{j}=\infty$ does not occur). We call $\left\{p_{1}, \ldots, p_{n-1}\right\}$ the Schläfli type of the polytope. If the polytope is determined just by the $p_{j}$, then we have the universal regular polytope (of that Schläfli type), for which we use the same symbol $\left\{p_{1}, \ldots, p_{n-1}\right\}$ (but without qualification); we write $\left[p_{1}, \ldots, p_{n-1}\right]$ for the corresponding Coxeter group. Generally, however, the group $\Gamma$ will satisfy additional relations as well, for some of which we shall introduce special notation later.

In the geometric context (that is, of realizations, for the general background to which see [15], [19] or Chapter 5 of [22]), each $\rho_{j}$ is represented (under some homomorphism) by a reflexion $R_{j}$ - an involutory isometry—which we identify with its mirror of fixed points

$$
\left\{x \mid x R_{j}=x\right\}
$$

We write $\mathcal{G}:=\left\langle R_{0}, \ldots, R_{n-1}\right\rangle$ for the corresponding symmetry group. The intersection property (2.1) translates directly into

$$
\left\langle R_{i} \mid i \in \mathrm{J}\right\rangle \cap\left\langle R_{i} \mid i \in \mathrm{K}\right\rangle=\left\langle R_{i} \mid i \in \mathrm{J} \cap \mathrm{K}\right\rangle .
$$

The structure of the realization $P$ is then given by Wythoff's construction, namely, the initial vertex is $F_{0}:=v \in R_{1} \cap \cdots \cap R_{n-1}$ (the latter is called the Wythoff space) and, recursively, the initial $j$-face is

$$
F_{j}:=\left\{F_{j-1} G \mid G \in\left\langle R_{0}, \ldots, R_{j-1}\right\rangle\right\}
$$


for $j=1, \ldots, n-1$; all other (proper) faces are images of the initial faces under $\mathcal{G}$. Further, $\Gamma_{-1}$ and $\Gamma_{n}$ are represented by $F_{-1}:=\emptyset$ and $F_{n}:=P$. The partial ordering of the faces is induced by iterated membership. The (affine) subspace

$$
E:=\operatorname{aff}\{v G \mid G \in \mathcal{G}\}
$$

spanned by the vertices of $P$ is called the ambient space of $P$; we lose no generality in thinking of this as the whole space in which we work. We then write $\operatorname{dim} P:=\operatorname{dim} E$, which is the dimension of $P$. Finally, the realization is faithful if $P$ (as a poset) is isomorphic to $\mathcal{P}$; it cannot be faithful if any $p_{j}=2$ (see [15] or Theorem 5A7 of [22]).

In the finite case, we can assume that $\mathcal{G}$ is an orthogonal group, and (to avoid trivialities) that $v \neq o$, the origin of coordinates.

There is no harm in adopting the view that an edge is the (line-)segment joining its two vertices, and that a 2 -face is the polygon determined by its constituent (vertices and) edges (we shall not need more than that here).

A realization of an abstract regular polytope $\mathcal{P}$ determines a realization of each of its faces or vertex-figures. In particular, $F_{n-1}$ (and its induced structure, with the same initial vertex $v$ ) gives a realization of the facet of $\mathcal{P}$; its symmetry group is the image $\mathcal{G}_{n-1}$ of $\Gamma_{n-1}$. If we write $w$ for the mid-point of the edge between $v$ and $v R_{0}$, then $w$ is the initial vertex of a realization of the vertex-figure of $\mathcal{P}$, with symmetry group the image $\mathcal{G}_{0}$ of $\Gamma_{0}$. Faithfulness is hereditary; that is, if the original realization of $\mathcal{P}$ is faithful, then the realizations of the facet and vertex-figure of $\mathcal{P}$ are also faithful.

We often find it more convenient to use $v R_{0}$ rather than $w$ as the initial vertex of the vertex-figure; for most purposes, this makes little difference, since the combinatorics are not altered.

There are important restrictions on faithful realizations, which we state only for the finite case (but in general terms); we refer to Sections 5B and 5C of [22] for proofs.

Theorem 2.1. Let $P$ be a faithful realization of a finite abstract regular polytope $\mathcal{P}$, whose ambient space $E$ is euclidean. Then $\operatorname{dim} P \geqslant \operatorname{rank} \mathcal{P}$.

Theorem 2.2. Let $P$ be a faithful realization of a finite abstract regular n-polytope in a euclidean space $E$, with group $\mathcal{G}=\left\langle R_{0}, \ldots, R_{n-1}\right\rangle$. Then $\operatorname{dim} R_{j} \geqslant j+1$ for $j=0, \ldots, n-2$, and $\operatorname{dim} R_{n-1} \geqslant n-1$.

The sequence ( $\left.\operatorname{dim} R_{0}, \operatorname{dim} R_{1}, \ldots, \operatorname{dim} R_{n-1}\right)$ is called the dimension vector of the realization. The first step in the classification of the faithfully realized regular polytopes of a fixed rank $n$ in a fixed dimension $d$ is to determine which dimension vectors can occur.

If we have (not necessarily faithful) realizations of the abstract regular polytope (or apeirotope) $\mathcal{P}$ in two euclidean spaces, say $P$ with mirrors $S_{0}, \ldots, S_{n-1}$ in $L$ and $Q$ with mirrors $T_{0}, \ldots, T_{n-1}$ in $M$ (possibly some $S_{j}=L$ or $T_{j}=M$ ), then their blend has mirrors $S_{j} \times T_{j}$ in $L \times M$ for $j=0, \ldots, n-1$. Indeed, if $v \in S_{1} \cap \cdots \cap S_{n-1}$ and $w \in T_{1} \cap \cdots \cap T_{n-1}$ are the initial vertices of the two realizations, then $(v, w)$ can be chosen as the initial vertex of the blend, which we then write $P \# Q$. A realization which cannot be expressed as a blend in a non-trivial way is called pure. 
We end the section with a general remark. Let $S$ and $T$ be linear reflexions. First, since $S T=(-S)(-T)=S^{\perp} T^{\perp}$ (thus identifying $-S$ with its mirror $S^{\perp}$, and so on), then $S \cap T$ and $S^{\perp} \cap T^{\perp}$ are both pointwise fixed by the product. That is, the axis (fixed set) of $S T$ is

$$
(S \cap T)+\left(S^{\perp} \cap T^{\perp}\right)=(S \cap T)+(S+T)^{\perp} .
$$

In particular, if $S$ and $T$ commute, then (2.2) is the mirror of their product $S T=T S$, which is again a reflexion.

\section{Operations on Polyhedra}

From now on, we confine our attention to regular polyhedra, that is, polytopes of rank 3 . Various operations provide ways of connecting regular polyhedra; in this section we discuss these operations and their implications.

Two different regular polyhedra (or, more generally, polytopes) may be related by what is called a mixing operation: the distinguished generators of the second group are certain products of those of the first (see Chapter 7 of [22]—we discuss the concept on an abstract level, but it has corresponding geometric implications for realizations). First, duality $\delta$ just reverses the order of the distinguished generators (and the order relation on the faces). It is worth noting that, in general, duals of faithfully realizable regular polytopes are not necessarily faithfully realizable at all (those of Petrials of the ordinary regular solids are particular examples), let alone in the same space.

Second, suppose that $\Gamma=\left\langle\rho_{0}, \rho_{1}, \rho_{2}\right\rangle$ is a string C-group, and consider the operation

$$
\left(\rho_{0}, \rho_{1}, \rho_{2}\right) \mapsto\left(\rho_{0} \rho_{2}, \rho_{1}, \rho_{2}\right)=:\left(\sigma_{0}, \sigma_{1}, \sigma_{2}\right) .
$$

We write $\Gamma^{\pi}:=\left\langle\sigma_{0}, \sigma_{1}, \sigma_{2}\right\rangle$, and we call $\Gamma \mapsto \Gamma^{\pi}$ the Petrie operation. The Petrie operation $\pi$ does not always yield a C-group, although such cases are rather exceptional, and we shall not meet any of them here. When it does, we implicitly take the Petrie operation to apply to the corresponding polyhedron as well, and so write $\mathcal{P} \mapsto \mathcal{P}^{\pi}$, which we call the Petrial of $\mathcal{P}$.

We note that an important class of regular polyhedra or apeirohedra consists of those which are determined by their Schläfli type and Petrie polygons. The geometric picture of a Petrie polygon is one which shares two successive edges of each 2-face which it meets, but not a third; thus the faces of $\mathcal{P}^{\pi}$ are the Petrie polygons of $\mathcal{P}$. We write $\{p, q\}_{r}$ for the polyhedron (possibly infinite) of Schläfli type $\{p, q\}$, whose Petrie polygons of length $r$ determine it. Its group is the Coxeter group $\left\langle\rho_{0}, \rho_{1}, \rho_{2}\right\rangle=[p, q]$, with the imposition of the single extra relation

$$
\left(\rho_{0} \rho_{1} \rho_{2}\right)^{r}=\varepsilon
$$

We note that, if it is a genuine polyhedron, then the Petrial of $\{p, q\}_{r}$ is $\{r, q\}_{p}$.

In the present context, we find it convenient to designate some regular polyhedra by the types of their faces, vertex-figures and Petrie polygons (in a more general sense which we describe in Section 5). Because the entries in the symbol can be fractions, the subscript notation is somewhat less than satisfactory. We therefore write $\{p, q: r\}$ for 
a regular polygon whose faces are polygons $\{p\}$, vertex-figures $\{q\}$ and Petrie polygons $\{r\}$; in the way that we use this notation, it will be clear that there are to be no implications of universality.

Third, suppose that $\mathcal{P}$ is a regular polyhedron of type $\{p, q\}$, and let $1<k<\frac{1}{2} q$, with greatest common divisor $(k, q)=1$. The facetting operation $\varphi_{k}$ is defined by

$$
\left(\rho_{0}, \rho_{1}, \rho_{2}\right) \mapsto\left(\rho_{0},\left(\rho_{1} \rho_{2}\right)^{k-1} \rho_{1}, \rho_{2}\right)=:\left(\sigma_{0}, \sigma_{1}, \sigma_{2}\right) .
$$

If $1<m<\frac{1}{2} q$ is such that $k m \equiv \pm 1 \bmod q$, then $\varphi_{m}$ inverts $\varphi_{k}$. Geometrically, the new polyhedron $\mathcal{P}^{\varphi_{k}}$ has the same vertices and edges as $\mathcal{P}$. However, successive edges of a 2 -face are now separated by $k$ steps along the old vertex-figure, instead of 1 (in some local orientation); the 2 -faces of $\mathcal{P}^{\varphi_{k}}$ are the $k$-holes of $\mathcal{P}$. In case $k=2$, we refer simply to the holes of $\mathcal{P}$.

The designation of a (possibly infinite) regular polyhedron of Schläfli type $\{p, q\}$, which is determined by its holes of length $h$, is $\{p, q \mid h\}$. The corresponding relation to be imposed on the Coxeter group $\left\langle\rho_{0}, \rho_{1}, \rho_{2}\right\rangle=[p, q]$ is

$$
\left(\rho_{0} \rho_{1} \rho_{2} \rho_{1}\right)^{h}=\varepsilon .
$$

Various examples of such polyhedra occur later; for now, let us observe that the great dodecahedron $\left\{5, \frac{5}{2}\right\}$ is, as an abstract regular polyhedron, $\{5,5 \mid 3\}$.

In practice, we sometimes use the notation $\{p, q \mid h\}$ merely to indicate the type of a regular polyhedron with faces $\{p\}$, vertex-figures $\{q\}$ and holes $\{h\}$. However, when we do this, some of the entries will be fractions, and so little confusion will arise.

Fourth, we have the halving operation $\eta$. This performs on the group $\Gamma=\left\langle\rho_{0}, \rho_{1}, \rho_{2}\right\rangle$ of a regular polyhedron $\mathcal{P}$ of type $\{4, q\}$ the operation

$$
\eta:\left(\rho_{0}, \rho_{1}, \rho_{2}\right) \mapsto\left(\rho_{0} \rho_{1} \rho_{0}, \rho_{2}, \rho_{1}\right)=:\left(\sigma_{0}, \sigma_{1}, \sigma_{2}\right) .
$$

This results (usually) in a self-dual polyhedron of type $\{q, q\}$, denoted by $\mathcal{P}^{\eta}$. It has half the vertices of $\mathcal{P}$ if all edge-circuits of $\mathcal{P}$ have even length; otherwise it has the same vertices. The group order (if finite) is halved just when the number of vertices is.

Our final operation is skewing (or skew halving) $\sigma$. It applies to a regular polyhedron of $\mathcal{P}$ type $\{p, 4\}$, and is defined by

$$
\sigma:\left(\rho_{0}, \rho_{1}, \rho_{2}\right) \mapsto\left(\rho_{1}, \rho_{0} \rho_{2},\left(\rho_{1} \rho_{2}\right)^{2}\right)=:\left(\tau_{0}, \tau_{1}, \tau_{2}\right) .
$$

It is remotely related to halving; in fact, as can be checked (see Section 7B of [22]),

$$
\sigma=\pi \delta \eta \pi \delta .
$$

This also indicates that $\sigma$ halves the order of the group just when $\eta$ does, modulo the double application of $\pi \delta$. If this is the case, then $\rho_{2} \notin \Gamma\left(\mathcal{P}^{\sigma}\right)$, but acts on it as an automorphism. In any event, $\mathcal{P}^{\sigma}$ is self-Petrie; the isomorphism between $\mathcal{P}^{\sigma}$ and $\mathcal{P}^{\sigma \pi}$ is induced by conjugation of $\Gamma\left(\mathcal{P}^{\sigma}\right)=\Gamma(\mathcal{P})^{\sigma}$ by $\rho_{2}$, since

$$
\rho_{2} \tau_{0} \rho_{2}=\rho_{2} \rho_{1} \rho_{2}=\rho_{1} \cdot\left(\rho_{1} \rho_{2}\right)^{2}=\tau_{0} \tau_{2} .
$$

It should perhaps be emphasized that all these operations really apply to groups (or their distinguished generators), rather than to polyhedra. This is particularly important in 
the case of $\sigma$ since, as we shall see in Section 11, we can use $\sigma$ to obtain non-degenerate polyhedra from degenerate ones.

One further abstract construction, which is a special case of mixing, is the following. Again, suppose that $\Gamma=\left\langle\rho_{0}, \rho_{1}, \rho_{2}\right\rangle$ is a string C-group. Let $\tau$ be an involution which commutes with all $\rho_{j}$, and consider the operation

$$
\left(\rho_{0}, \rho_{1}, \rho_{2}, \tau\right) \mapsto\left(\rho_{0} \tau, \rho_{1}, \rho_{2}\right)=:\left(\sigma_{0}, \sigma_{1}, \sigma_{2}\right) .
$$

This is called mixing with a segment, because $\tau$ can be regarded as the generating involution of the group of the segment (1-polyhedron) \{\} . We have (see Theorem 7A8 of [22])

Theorem 3.1. Mixing a string $C$-group $\Gamma$ with a segment always yields another $C$ group. This is isomorphic to $\Gamma$ if all edge-circuits in the associated regular polyhedron $\mathcal{P}$ have even length; otherwise, it is isomorphic to the direct product $\Gamma \times \mathcal{C}_{2}$ of $\Gamma$ with a cyclic group $\mathcal{C}_{2}$ of order 2 .

We denote the resulting regular polyhedron (which again we say is obtained from $\mathcal{P}$ by mixing with a segment) by $\mathcal{P} \diamond\{\}$. This has twice as many vertices as $\mathcal{P}$ precisely when some edge-circuit of $\mathcal{P}$ has odd length.

If $P$ is a realization of the abstract regular polytope $\mathcal{P}$ with vertex-set $V$, then the mix $\mathcal{P} \diamond\{\}$ admits a realization whose vertices are a subset of $V \times\{ \pm 1\}$. However, in some cases, there may be a realization of $\mathcal{P} \diamond\{\}$ with the same ambient space as $P$.

Another technique which we use here is twisting (see Chapter 8 of [22]). In this, a given group generated by involutions (usually itself a C-group) is augmented by means of one or more automorphisms. Although it is central to our classification, we shall not go into this any further here; we shall see various applications in the appropriate places.

\section{Dimension Vectors}

As a first step in describing which geometrically realized polyhedra $P$ occur in $\mathbb{E}^{4}$, we need to determine the possible dimension vectors $\left(\operatorname{dim} R_{0}, \operatorname{dim} R_{1}, \operatorname{dim} R_{2}\right)$ of the mirrors $R_{0}, R_{1}, R_{2}$ of the reflexions which generate the corresponding symmetry groups $\mathcal{G}(P)$. Theorem 2.2 provides a starting point; the dimension vector must satisfy

$$
(1,2,2) \leqq\left(\operatorname{dim} R_{0}, \operatorname{dim} R_{1}, \operatorname{dim} R_{2}\right) \leqq(3,3,3),
$$

where $\leqq$ indicates inequality in each component individually.

We now proceed as follows. Using essentially the same trick as in the earlier papers [18], [21], if the mirror $R_{0}$ satisfies $\operatorname{dim} R_{0}=1$, then we can replace it by

$$
-R_{0}=R_{0}^{\perp},
$$

its orthogonal complement, which (as an isometry) is its product with the central inversion $-I$; we denote this general operation by $\kappa$. (In [18] we called this operation $\kappa_{0}$, but here no more general operations $\kappa_{i j}$ or $\kappa_{j}$ are needed.) We always obtain another finite group 
$\mathcal{G}^{\prime}$; in fact,

$$
\left|\mathcal{G}^{\prime}\right|=\frac{1}{2}|\mathcal{G}|,|\mathcal{G}| \text {, or } 2|\mathcal{G}| \text {. }
$$

It will turn out that we always obtain another regular polyhedron as well.

Next, if $\operatorname{dim} R_{0}=2$ and $\operatorname{dim} R_{2}=3$ (or vice versa, but we shall see that this case will have to be excluded), then we can replace $R_{0}$ by $R_{0} R_{2}$, that is, apply (or reverse) the Petrie operation $\pi$; bearing in mind (2.2), the new $R_{0}$ has $\operatorname{dim} R_{0}=1$ or 3, and in the former case we can proceed as previously.

Finally, as long as our (possibly new) group contains a hyperplane reflexion (that is, $\operatorname{dim} R_{j}=3$ for some $j$ ), we can regard $\mathcal{G}$ as a reflexion (Coxeter) group, on which certain involutions with two-dimensional mirrors act as automorphisms (more precisely, $\mathcal{G}$ is the corresponding semi-direct product). When we have carried out the foregoing procedures, it will later become clear that only those polyhedra whose groups have dimension vectors $(3,2,3)$ or $(2,3,2)$ need to be analysed in detail. For classification purposes, we then reverse the procedure: the starting point is a Coxeter group, not necessarily with standard generators, which can be represented by a diagram that permits permutation of its nodes.

The one case which is not covered by the previous analysis is dimension vector $(2,2,2)$. This class is closed under the standard operations $\pi$ and $\kappa$; as we shall see, duality does not apply here. In fact, there are polyhedra in this class whose symmetry groups are not subgroups of reflexion groups. The approach here will be through quaternions, which will enable us to relate such a polyhedron to a pair of (sometimes degenerate) polyhedra in $\mathbb{E}^{3}$ or, rather, the corresponding regular projective polyhedra.

We now briefly analyse the possibilities in general terms, leaving the details and the enumeration problems for each class until subsequent sections. In the listing of the classes, we group together those which are related by Petriality $\pi$ or the operation $\kappa$; as we have already remarked, duality $\delta$ is not always applicable.

- $(3,3,3)$. For this case, the intersection $R_{0} \cap R_{1} \cap R_{2}$ of three linear hyperplanes will be a line, and so a corresponding polyhedron will only be three-dimensional. It must therefore be excluded (but only on these grounds).

- $(1,3,3)$. This case is allowed; $\kappa$ can be applied to the (suitably realized) case $(3,3,3)$. We shall see in Section 6 that these polyhedra can also be regarded as blends, with one component degenerating to a segment.

- $(2,3,3)$. An appeal to (2.2) shows that applying Petriality $\pi$ to this case yields either case $(3,3,3)$ or case $(1,3,3)$. The first possibility must be excluded on the same grounds as before, but the second will occur.

- $(3,3,2)$. This would be the dual class to $(2,3,3)$. However, even in the allowed case (the Petrials of class $(1,3,3)$ ), the faces of the original polyhedron are centred at $o$, and so the dual must be excluded.

- $(1,3,2)$. If this class occurred, it would be obtained from the class $(3,3,2)$ by applying $\kappa$; we must therefore disallow it.

- $(3,2,3)$. Let the group be $\mathcal{G}=\left\langle R_{0}, R_{1}, R_{2}\right\rangle$. Then $R_{1}$ acts as an automorphism on the hyperplane reflexion group $\mathcal{H}=\left\langle S_{0}, \ldots, S_{3}\right\rangle$, given by

$$
\left(S_{0}, \ldots, S_{3}\right):=\left(R_{0}, R_{1} R_{2} R_{1}, R_{2}, R_{1} R_{0} R_{1}\right) .
$$

The underlying diagram of $\mathcal{H}$ is denoted $\mathcal{D}_{1}(p, q ; r)$ as in Fig. 1 (the notation indicates that $p$ and $q$ play a similar rôle, while that of $r$ is different). The labels on 


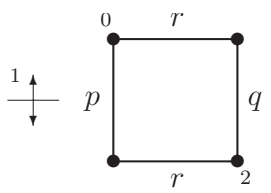

Fig. 1. The diagram $\mathcal{D}_{1}(p, q ; r)$.

the nodes of the diagram indicate the reflexions $R_{0}$ and $R_{2}$, while $R_{1}$ is indicated by the implied flip of the diagram. Moreover, we must have $p, q>1$, where $p$ is not a fraction with an even denominator (we explain why in Section 7) and $r>2$; the resulting polyhedron is of type $\{2 p, 2 q \mid r\}$. In case $q$ is also not a fraction with an even denominator, then duality $\delta$ interchanges $p$ and $q$. There is an alternative approach when $q$ is a fraction with an even denominator; in this event, the automorphism $R_{1}$ of the diagram is inner, and the group of the polyhedron can be obtained from that of a regular 4-polytope by a mixing operation.

- $(1,2,3)$. This case arises from the case $(3,2,3)$ by applying $\kappa$.

- $(2,2,3)$. This case is obtained from either case $(3,2,3)$ or case $(1,2,3)$ by Petriality $\pi$; naturally, the two possibilities have to be distinguished. These three classes of polyhedra (or four if we make the distinction implied in the last sentence) fall into two families with the same vertices.

- $(3,2,2)$. This case would arise from $(2,2,3)$ by duality. However, it may be seen that, whichever way the original group of type $(2,2,3)$ arises, the product $R_{0} R_{1}$ of its corresponding reflexions $R_{0}$ and $R_{1}$ is a double rotation (in two orthogonal planes), since $R_{0} \cap R_{1}=\{o\}$; it follows that the class cannot occur.

- $(1,2,2)$. This case would be obtained from $(3,2,2)$ by applying $\kappa$, and so it too must be excluded.

- $(2,3,2)$. This class gives rise to rich families. In this case, $R_{0}$ and $R_{2}$ are the automorphisms, which act on a diagram given by

$$
\left(S_{0}, \ldots, S_{3}\right):=\left(R_{1}, R_{0} R_{1} R_{0}, R_{0} R_{2} R_{1} R_{2} R_{0}, R_{2} R_{1} R_{2}\right)
$$

The general case is thus derived from a diagram $\mathcal{D}_{2}(p, q, r)$, as in Fig. 2. Once again, the labels on the nodes and implied automorphisms correspond to the original reflexions. The resulting polyhedron is of type $\{2 p, 2 q: 2 r\}$, from which are obtained up to five others by duality $\delta$ and Petriality $\pi$; observe that these two operations preserve the class $(2,3,2)$. We have $p, q, r>1$, but $q$ must not be a fraction with an even denominator; we shall see why in Section 8.

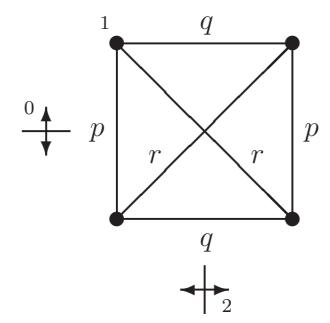

Fig. 2. The diagram $\mathcal{D}_{2}(p, q, r)$. 
- $(2,2,2)$. This is the anomalous case, to which the notion of a Coxeter group with outer automorphisms is inapplicable. Indeed, some examples of this kind cannot be related to Coxeter groups in any meaningful way. The approach here is through quaternions; see Section 10.

Remark 4.1. Lowering each entry of these dimension vectors by one gives a potential dimension vector for polyhedra or apeirohedra in $\mathbb{E}^{3}$. If we reinstate $(3,3,3)$ (which yields only three-dimensional polyhedra), then exactly the analogues of those which give polyhedra in $\mathbb{E}^{4}$ are valid dimension vectors in $\mathbb{E}^{3}$.

\section{Notation}

One aim in this section is to introduce an abbreviated notation for regular polygons, with a view to providing a description of regular polyhedra in subsequent sections which captures at least some of their geometry. We also need to discuss which kinds of polygons are generated by a given pair of reflexions in $\mathbb{E}^{4}$. We have already introduced notations which indicate the holes or Petrie polygons of a polyhedron as well.

Referring to the analysis of [15] or Section 5B of [22], there can be three types of regular polygons in $\mathbb{E}^{4}$. We exclude the digon \{\} (a degenerate polygon, collapsing onto a segment); however, \{\} can be a component of a regular polygon in a blend, and in that context should be thought of as an abbreviation for $\{2\}$.

First, we have a planar polygon $\{p\}$, with $p>2$ a rational number. If $p=s / t$ in lowest terms, then $\{p\}=\left\{\frac{s}{t}\right\}$ is a regular $s$-gon, whose edges subtend an angle $2 \pi t / s$ at its centre. If $t=1$, we just write $\{p\}$ rather than $\left\{\frac{p}{1}\right\}$; in what follows, though, we must sometimes bear in mind this suppressed denominator "1".

Second, we have a three-dimensional skew polygon or zigzag. This will always be a $2 s$-gon for some $s$, and will be denoted by $\left\{\frac{2 s}{t, s}\right\}$ for some $1 \leqslant t<s$; here, the greatest common divisor of $2 s$ and $t$ is $(2 s, t)=1$ or 2 . It will be a blend of a segment \{\}$=\{2\}$ with a planar regular polygon, which will be $\left\{\frac{2 s}{t}\right\}$ if $t$ is odd, or $\left\{\frac{s}{t / 2}\right\}$ if $t$ is even.

Third, we have a four-dimensional helical polygon or helix, whose notation is $\left\{\frac{p}{s, t}\right\}$. This is a blend of planar regular polygons $\left\{\frac{p^{\prime}}{s^{\prime}}\right\}$ and $\left\{\frac{p^{\prime \prime}}{t^{\prime}}\right\}$, where $p^{\prime} / s^{\prime}=p / s, p^{\prime \prime} / t^{\prime \prime}=$ $p / t$, and $p$ is the least common multiple of $p^{\prime}$ and $p^{\prime \prime}$ (thus the greatest common divisor $(p, s, t)=1)$. Conventionally, we assume that $s<t$, so that $1 \leqslant s<t<\frac{1}{2} p$. If, in our subsequent calculations, a mark $t>\frac{1}{2} p$ occurs, it should be replaced by $p-t$ (geometrically, the polygons are the same, but the orientation will be different, if we cared to keep track of it).

Now let $S$ and $T$ be mirrors of (linear) reflexions in $\mathbb{E}^{4}$. We take a point $v \in T \backslash S$, and consider the regular polygon $P$ (assumed finite) with $v$ as the initial vertex, and $S$ and $T$ playing the rôles of $R_{0}$ and $R_{1}$. For reasons made clear in Section 4, we suppose that $\operatorname{dim} S \geqslant 1$ and $\operatorname{dim} T \geqslant 2$. To ensure non-degeneracy, neither of $S$ or $T$ is a subspace of the other; moreover, as isometries, $S T \neq T S$.

Case 1: $(\operatorname{dim} S, \operatorname{dim} T)=(3,3)$. Here, $P$ is planar, with centre on the two-dimensional axis $S \cap T$. 
Case 2: $(\operatorname{dim} S, \operatorname{dim} T)=(2,3)$. We must have $\operatorname{dim}(S \cap T)=1$; in general, $P$ will be skew.

Case 3: $(\operatorname{dim} S, \operatorname{dim} T)=(3,2)$. Here, $P$ will again be planar.

Case 4: $(\operatorname{dim} S, \operatorname{dim} T)=(2,2)$. In this case, $P$ will be planar or helical, according as $\operatorname{dim}(S \cap T)=1$ or 0 .

Case 5: $(\operatorname{dim} S, \operatorname{dim} T)=(1,3)$. Since this is obtained from Case 1 by an application of $\kappa$, we see that, in general, $P$ will be skew.

Case 6: $(\operatorname{dim} S, \operatorname{dim} T)=(1,2)$. Once again, $P$ will be skew; indeed, $P$ will lie in the subspace $\operatorname{lin}(S \cup T)$ of $\mathbb{E}^{4}$.

\section{Blended Polyhedra}

In the case of regular polyhedra, the only blended realizations which can occur are those obtained from a three-dimensional polyhedron by mixing with a segment. The two components (the original polyhedron and the segment) are then realizations of the four-dimensional polyhedron, degenerate in the second case, and possibly also in the first.

We noted in Section 4 that the dimension vector $(3,3,3)$ has to be excluded, because the resulting regular polyhedra are only three-dimensional. However, we can realize a regular polyhedron $Q$ with centre $o$ in $\mathbb{E}^{3}$ (obtained from a group of type $(2,2,2)$ ) as $Q \times\{1\}$ in the hyperplane $H:=\mathbb{E}^{3} \times\{1\}$; applying $\kappa$ then yields a polyhedron $P$ whose vertices lie in $H$ or in the reflected hyperplane $-H$. Exactly the same procedure applies to the Petrials of such polyhedra $Q$, with dimension vectors $(1,2,2)$.

Abstractly, $P=Q \diamond\{\}$, the mix of $Q$ with a segment \{\} . Now $P \cong Q$ exactly when every edge-circuit of $Q$ is even; the only two three-dimensional regular polyhedra $Q$ for which this is the case are the cube $\{4,3\}$ and its Petrial $\{6,3: 4\}=\{6,3\}_{4}$. Alternate vertices (in edge-circuits of $Q$ with an arbitrary starting point) are then displaced from $H$ to $-H$.

For each of the 16 others (in Petrie pairs), the vertices are those of the cartesian product $Q \times\{\}$. In more detail, if $v, w$ are vertices of $Q$ which are joined by an edge, then there is a corresponding edge in $P=Q^{\kappa}$ which joins $v$ and $-w$. As we have remarked, an edge-circuit in $P$ is doubled in length from the corresponding one in $Q$ precisely when its length is odd.

It is clear that, as a realization, $P$ will be blended. One component is obviously the segment \{\} , which admits a natural covering by $P$ (since all edge-circuits of $P$ have even length, the edge-graph of $P$ has a 2-colouring). It might be thought that the other component would be $Q$, with a corresponding covering $P \searrow Q$ of $Q$ by $P$. However, we have already observed in [21] that the operation $\kappa^{\prime}$ (analogous to $\kappa$ ) on regular 3polytopes pairs up the classical polyhedra and their Petrials in a different way from Petriality. This alternative pairing $Q \leftrightarrow Q^{\prime}:=Q^{\kappa^{\prime}}$ (as 3-polytopes) then results in the identification $Q \diamond\{\}=Q^{\prime} \#\{\}$. 
Our usual convention identifies a component of a blend with the polytope which is covered (we shall not encounter cases when the covered object is only a pre-polytope, in the terminology of Section 2D of [22]). We can now list the nine four-dimensional regular polyhedra whose groups have dimension vectors $(1,3,3)$, with the pairings given by mixing (which we realize here by $\kappa$ ) and blending:

$$
\begin{aligned}
& \{3,3\} \diamond\{\}=\left\{\frac{6}{1,3}, 3: 4\right\} \#\{\}, \\
& \{3,4\} \diamond\{\}=\left\{\frac{6}{1,3}, 4: 3\right\} \#\{\}, \\
& \{4,3\} \diamond\{\}=\left\{\frac{4}{1,2}, 3: 3\right\} \#\{\}, \\
& \{5,3\} \diamond\{\}=\left\{\frac{10}{3,5}, 3: 5\right\} \#\{\}, \\
& \{3,5\} \diamond\{\}=\left\{\frac{6}{1,3}, 5: \frac{5}{2}\right\} \#\{\}, \\
& \left\{5, \frac{5}{2}\right\} \diamond\{\}=\left\{\frac{10}{3,5}, \frac{5}{2}: 3\right\} \#\{\}, \\
& \left\{\frac{5}{2}, 5\right\} \diamond\{\}=\left\{\frac{10}{1,5}, 5: 3\right\} \#\{\}, \\
& \left\{3, \frac{5}{2}\right\} \diamond\{\}=\left\{\frac{6}{1,3}, \frac{5}{2}: 5\right\} \#\{\}, \\
& \left\{\frac{5}{2}, 3\right\} \diamond\{\}=\left\{\frac{10}{1,5}, 3: \frac{5}{2}\right\} \#\{\} .
\end{aligned}
$$

The notation for the polyhedra in the second column indicates their types of faces and Petrie polygons, as explained in Section 5.

The class $(2,3,3)$ is obtained from the class $(1,3,3)$ by Petriality. We get the polyhedra in this class by interchanging the mix $(\kappa)$ and blend in the list for the class $(1,3,3)$. This gives an additional nine polyhedra.

These polyhedra do not admit dual classes $(3,3,1)$ (which is excluded on general grounds) or $(3,3,2)$. In the second case, though the faces of the polyhedra in the class $(2,3,3)$ are only three-dimensional, their centres are at the origin $o$, and so the "duals" degenerate.

\section{Dimension Vector $(3,2,3)$ and Its Relatives}

In this section we consider the regular polyhedra with dimension vector $(3,2,3)$; we say rather less about those which are derived from them using the operations $\kappa, \pi$ or both. Where appropriate, though, we look at the effect of the operations $\varphi_{k}$; however, we leave discussion of $\eta$ and $\sigma$ until Section 11.

Let the group of a polyhedron in this class be $\mathcal{G}=\left\langle R_{0}, R_{1}, R_{2}\right\rangle$, and consider the operation

$$
\left(R_{0}, R_{1}, R_{2}\right) \mapsto\left(R_{0}, R_{1} R_{0} R_{1}, R_{2}, R_{1} R_{2} R_{1}\right)=:\left(S_{0}, S_{1}, S_{2}, S_{3}\right) ;
$$

each of the $S_{j}$ is now a hyperplane reflexion. Since $R_{0}$ and $R_{2}$ commute, the picture 
here-as we saw in Section 4-is of a (generalized) Coxeter diagram of the form

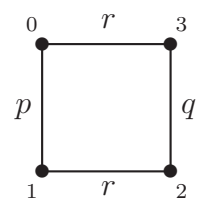

The label $j$ on a node corresponds to the generator $S_{j}$ of the reflexion subgroup $\mathcal{H}:=$ $\left\langle S_{0}, S_{1}, S_{2}, S_{3}\right\rangle$. For geometric reasons, we cannot have $r=2$; otherwise, $\left\langle S_{0}, S_{1}\right\rangle$ and $\left\langle S_{2}, S_{3}\right\rangle$ act on orthogonally complementary planes of $\mathbb{E}^{4}$, and the corresponding "polyhedron" degenerates to a polygon (or, more strictly perhaps, a dihedron). However, $p=2$ or $q=2$ (or both) are allowed. Observe that any of the marks $p, q$ or $r$ may be fractional (with a restriction on $p$ which will be explained shortly); in other words, the mark $p$ indicates the angle $\pi / p$ between the mirrors $S_{0}$ and $S_{1}$, and similarly for $q$ or $r$, and so we only demand that the marks $p$ and $q$ be greater than 1 .

Let us go a little deeper here into the geometric detail. The mirrors $R_{0}$ and $R_{1}$ whose reflexions generate the group of the initial 2-face are of dimensions 3 and 2, respectively, and so (generically) their intersection has dimension 1 . The initial vertex lies in $R_{1}$ but not in $R_{0}$, and so its images under $\mathcal{G}_{2}=\left\langle R_{0}, R_{1}\right\rangle$ will only span a plane; that is, the 2 -faces of polyhedra in this class are planar. However, for the vertex-figure, the situation is reversed: $R_{1}$ has dimension 2 while $R_{2}$ has dimension 3 , and so the vertex-figure will be (in general) a skew (three-dimensional) polygon. With the notation of the diagram, the faces will thus be planar of type $\{2 p\}$, with the appropriate interpretation when $p$ is fractional. We thus see at once that $p=s / t$ cannot be a fraction with an even denominator $t$, because then the resulting polygon doubly covers $\left\{\frac{s}{t / 2}\right\}$; this restriction is that mentioned in Section 4.

In general, the faces of the resulting polyhedron are the vertices, edges and (planar) faces

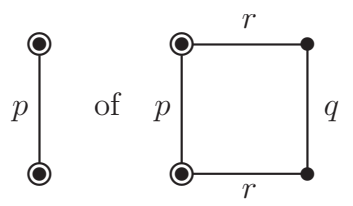

Moreover, the remaining 2-faces

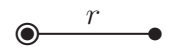

of the 4-polytope form holes in the polyhedron. Finally, the vertex-figure is a skew polygon; if $q=s / t$, then it is in fact $\left\{\frac{2 s}{t, s}\right\}$.

For the enumeration, we begin with the case $p=q=2$; any $r>2$ is allowed. The corresponding diagram is
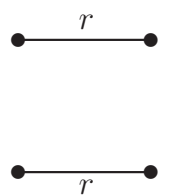
with the horizontal flip understood. We obtain a toroid which we can designate

$$
\left\{4, \frac{4}{1,2} \mid r\right\}
$$

even when $r$ is fractional; it is isomorphic to the universal toroid $\{4,4 \mid s\}=\{4,4\}_{(s, 0)}$ when $r=s / t$ in its lowest terms, and its group is $\left(D_{s} \times D_{s}\right) \rtimes C_{2}$ (meaning an extension of the product of two copies of the dihedral group $D_{s}$ by an outer automorphism of order 2), and has order $2 \cdot(2 s)^{2}=8 s^{2}$. The geometric description is straightforward: take the cartesian product $\{r\} \times\{r\}$ of two polygons $\{r\}$, bearing in mind that $r$ can be a fraction (so that $\{r\}$ would then be a star-polygon). The vertices and edges are those of the product, while the square faces $\{4\}$ are the products of edges, one from each component; the vertex-figure is a skew square $\left\{\frac{4}{1,2}\right\}$. Note that this polyhedron is self-dual. (See also [24] for further realizations of toroids.)

Henceforth, then, we can assume that at least one of $p$ or $q$ exceeds 2 , so that the diagram is connected. It thus follows that the corresponding Coxeter group will be irreducible. One approach is to look through suitable presentations of such Coxeter groups (that is, Coxeter diagrams with, possibly, fractional marks-Goursat tetrahedra in terms of Section 14.8 of [4]) which admit appropriate automorphisms.

Our first examples derive from the diagram of the extended group $[3,3,3] \rtimes C_{2}$ of the regular simplex (again, the horizontal flip is understood, with the extension of the group $[3,3,3]$ by an outer automorphism of order 2 as before):

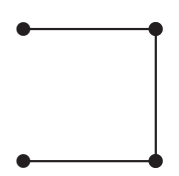

There result two polyhedra: the universal $\{4,6 \mid 3\}$ and its dual $\{6,4 \mid 3\}$. These were first described by Coxeter in [3]. In our more geometrical notation, they are

$$
\left\{4, \frac{6}{1,3} \mid 6\right\}, \quad\left\{6, \frac{4}{1,2} \mid 4\right\} .
$$

From the extended group $[3,4,3] \rtimes C_{2}$ of the regular 24-cell, we derive the diagrams
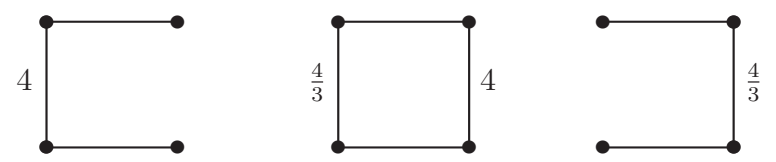

each of which permits a top-to-bottom flip, and thereby gives two dual regular polyhedra with dimension vectors $(3,2,3)$. From the first diagram, we obtain the universal dual regular polyhedra $\{4,8 \mid 3\}$ and $\{8,4 \mid 3\}$ of [3]. In the notation which we introduced in Section 5, the six resulting polyhedra are

$$
\left\{8, \frac{4}{1,2} \mid 3\right\}, \quad\left\{4, \frac{8}{1,4} \mid 3\right\}, \quad\left\{8, \frac{8}{3,4} \mid 3\right\}, \quad\left\{\frac{8}{3}, \frac{8}{1,4} \mid 3\right\}, \quad\left\{4, \frac{8}{3,4} \mid 3\right\}, \quad\left\{\frac{8}{3}, \frac{4}{1,2} \mid 3\right\} ;
$$

bear in mind here that the notation is only intended to indicate the geometry of the faces, vertex-figures and holes. These polyhedra are related alternately by duality $\delta$ and 
the facetting operation $\varphi_{3}$ (because the index $k=3$ in $\varphi_{k}$ is odd, the dimension vector $(3,2,3)$ is preserved).

We now need to comment on the remaining possibilities. As we have said, we can exclude the possibility that $p$ is a fraction with an even denominator. Leaving aside, for the moment, the case when $q$ is a fraction with an even denominator, this denies any further examples arising from the two groups already considered, or from the remaining Coxeter groups apart from [3, 3, 5]. However, among the Goursat tetrahedra in this last group is

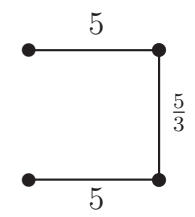

This diagram apparently fulfils all the required conditions. In fact, the automorphism here is inner, because the group $[3,3,5]$ is not a proper subgroup of any finite orthogonal group in $\mathrm{O}_{4}$; therefore, it does not lead to a new regular polyhedron, because the realization is degenerate. More precisely, if the polyhedron existed, then its vertices and edges would be (as we have observed) those of

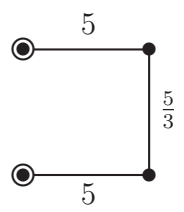

From the symmetry, the $14400 / 10=1440$ vertices would lie in pairs in the (possibly extended) edges of the 600 -cell $\{3,3,5\}$. However, direct calculation shows that the initial vertex is actually one of $\{3,3,5\}$, and so the vertices coincide in twelves with those of $\{3,3,5\}$. Similar considerations apply to other putative examples.

There remain the cases when $q$ is a fraction with an even denominator (these were overlooked in [23]). In Equation (10) in Section 6.1 of [20], it was observed that the mixing operation

$$
\left(T_{0}, \ldots, T_{3}\right) \mapsto\left(T_{1}, T_{0} T_{2}, T_{3}\right)=:\left(R_{0}, R_{1}, R_{2}\right)
$$

on the group $\left\langle T_{0}, \ldots, T_{3}\right\rangle$ of a regular 4-polytope $Q$ yields that of a regular polyhedron $P$. With one exception, $P$ has the following description. The edges of its faces join successive mid-points of edges of Petrie polygons of the facets of $Q$; the faces can then be thought of as central sections of these facets (this is actually the case when $Q$ is convex). If $Q=\left\{q_{1}, q_{2}, q_{3}\right\}$, with $q_{3}=s / t \neq 4$ (this is the exception), then the vertexfigure is $\left\{\frac{2 s}{t, s}\right\}$; note that each edge of a facet of $Q$ belongs to two of its Petrie polygons. In case $Q=\{3,3,4\}$, the resulting "polyhedron" splits into three copies of the torus $\{4,4 \mid 4\}$. 
We thus obtain 15 regular polyhedra, the generating reflexions of whose groups have dimension vector (3, 2, 3). Applying the operations (7.2) and (7.1) in turn yields

$$
\left(T_{0}, \ldots, T_{3}\right) \mapsto\left(T_{1}, T_{0} T_{2} T_{1} T_{0} T_{2}, T_{3}, T_{2} T_{3} T_{2}\right),
$$

which corresponds to the diagram $\mathcal{D}_{1}(p, q ; r)$, where

- $p=\frac{1}{2} h$, with $\{h\}$ the Petrie polygon of the facet $\left\{q_{1}, q_{2}\right\}$ of $Q$ (strictly speaking, the Petrie polygon is skew, and $p$ corresponds to the planar polygon whose basic rotation is $\left.\left(T_{0} T_{2} T_{1}\right)^{2}\right)$;

- $q=\frac{1}{2} q_{3}$

- $\{r\}$ is the hole of the vertex-figure $\left\{q_{2}, q_{3}\right\}$ of $Q$, namely, the face of $\left\{q_{2}, q_{3}\right\}^{\varphi_{2}}$.

We may also observe that we can recover the original 4-polytope $Q$ from the diagram $\mathcal{D}_{1}(p, q ; r)$ :

- $q_{3}=2 q$;

- $q_{3}$ and $r$ yield $q_{2}$-indeed, $r=t$ for $\{3, t\}$ and $\{t, 3\}$, while $r=3$ for $\left\{5, \frac{5}{2}\right\}$ and $\left\{\frac{5}{2}, 5\right\}$;

- $q_{2}$ and $h=2 p$ then give $q_{1}$ by

$$
\cos ^{2} \frac{\pi}{q_{1}}=\cos ^{2} \frac{\pi}{h}-\cos ^{2} \frac{\pi}{q_{2}}
$$

(compare equation 2.33 of [4]). The corresponding (abstract) groups of the regular star-polytopes which occur here are described in [17].

Of course, because $q$ is a fraction with an even denominator ( $q_{3}$ is odd), none of these 15 polyhedra has a realizable dual in $\mathbb{E}^{4}$.

Let us illustrate this latter discussion with a couple of examples:

$$
\begin{array}{ll}
\left\{3,5, \frac{5}{2}\right\} \mapsto\left\{10, \frac{10}{4,5} \mid 3\right\}, & \text { with diagram } \mathcal{D}_{1}\left(5, \frac{5}{4} ; 3\right), \\
\left\{\frac{5}{2}, 3,5\right\} \mapsto\left\{\frac{10}{3}, \frac{10}{2,5} \mid 5\right\}, & \text { with diagram } \mathcal{D}_{1}\left(\frac{5}{3}, \frac{5}{2} ; 5\right) .
\end{array}
$$

We say little about the related classes $(1,2,3)$ and $(2,2,3)$, which are derived from the class $(3,2,3)$ by $\kappa$, Petriality $\pi$, or both, except to remark that they contribute to the final classification. It should be observed (as the analysis of Section 4 shows) that none of these derived polyhedra can have geometrically realizable duals in $\mathbb{E}^{4}$. For example, the faces of the Petrial of $\left\{4, \frac{4}{1,2} \mid r\right\}$ are helices of type $\left\{\frac{2 s}{t, s-t}\right\}$ (if $r=s / t$ as before); all have the same vertex-figure $\left\{\frac{4}{1,2}\right\}$.

Perhaps, though, it is worth briefly remarking on the types of the Petrie polygons of the polyhedra with group $[3,4,3] \rtimes C_{2}$. Referring to the previous list, they are, respectively, $\left\{\frac{24}{1,11}\right\}$ for the first two $\left\{\frac{12}{1,5}\right\}$ for the next two and $\left\{\frac{24}{5,11}\right\}$ for the last.

Note that $\kappa$ interchanges polyhedra in the class $(2,2,3)$ derived from the class $(3,2,3)$ with those derived from $(1,2,3)=(3,2,3)^{\pi}$ (in an obvious informal notation). In summary, we thus derive a further $3 \cdot 23=69$ regular polyhedra, apart from infinitely many derived from the toroids, to add to the $8+15=23$ listed previously. 


\section{Dimension Vector $(2,3,2)$}

As we saw in Section 4, a polyhedron in the class $(2,3,2)$ is derived from a diagram of the form

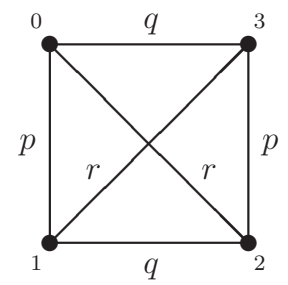

Thus, in this case, $R_{0}$ and $R_{2}$ are automorphisms which act on a diagram given by

$$
\left(S_{0}, \ldots, S_{3}\right):=\left(R_{1}, R_{0} R_{1} R_{0}, R_{0} R_{2} R_{1} R_{2} R_{0}, R_{2} R_{1} R_{2}\right) .
$$

The labels on the nodes now correspond to the new (hyperplane) reflexions $S_{j}$.

Again, as we have already indicated, the resulting polyhedron is of type $\{2 p, 2 q: 2 r\}$ (we explain how to interpret these numbers below), from which are obtained up to five others by duality $\delta$ and Petriality $\pi$. Note that the three operations $\delta, \pi$ and $\kappa$ preserve the class $(2,3,2)$; indeed, we have

$$
\delta: p \leftrightarrow q, \quad \pi: p \leftrightarrow r, \quad \kappa: p \leftrightarrow p^{\prime}, r \leftrightarrow r^{\prime},
$$

where

$$
\frac{1}{p}+\frac{1}{p^{\prime}}=1, \quad \frac{1}{r}+\frac{1}{r^{\prime}}=1 .
$$

Applying the same analysis as in Section 7, we see that the 2-faces of the polyhedron are skew $(2 p)$-gons of the form $\{p\} \#\{\}$. Here, it does not matter if $p$ is a fraction with an even denominator; the skew nature of the face prevents a collapse. However, the vertex-figure is a planar polygon so that, if $q$ is a fraction with an even denominator, then collapse occurs and the polyhedron degenerates. This is the restriction on $q$ which we mentioned in Section 4.

Notice that $R_{0} R_{2}$ is also an automorphism of the diagram. For non-degeneracy, it turns out that at least one of $R_{0}, R_{2}, R_{0} R_{2}$ must be an outer automorphism; in general, then, two of them will be outer and the other inner.

Bearing these considerations in mind, we readily see that the only diagrams of the above form which admit suitable automorphisms are given by the following unordered triples $\{p, q, r\}$ - which should not be confused with Schläfli symbols of regular 4polytopes (none actually is such a symbol). First, $\{p, 2,2\}$ (with an arbitrary fractional $p>2$, except that such a fraction with an even denominator cannot play the rôle of $q$ ); second, $\left\{3,3, \frac{3}{2}\right\},\left\{4,4, \frac{3}{2}\right\},\left\{\frac{4}{3}, \frac{4}{3}, \frac{3}{2}\right\}$ (in these three we cannot have $q=\frac{3}{2}$, and so each gives only two polyhedra); third, $\left\{3,4, \frac{4}{3}\right\}$ (here, any permutation is allowed, and so we obtain a full family of six). Here, the group [3, 3, 5] makes no contribution, even though (for instance) we do have a Goursat tetrahedron corresponding to $\mathcal{D}_{2}\left(3,5, \frac{5}{3}\right)$. As we observed in Section 7, [3, 3,5] has no outer automorphisms as a subgroup of $\mathrm{O}_{4}$, and we shall see in Section 11 why the remaining possiblilities must also be eliminated. 
We briefly discuss these polyhedra. In the first case, the two automorphisms (symmetries) $R_{2}$ and $R_{0} R_{2}$ of the diagram

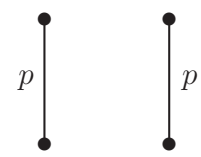

are both outer; their composition $R_{0}$ is also an outer automorphism, except when $p$ is a fraction with an even denominator. (Note that $R_{2}$ and $R_{0} R_{2}$ give the two involutory ways of interchanging the two dihedral subdiagrams.) In any event, if $p=s / t$ (in lowest terms), then the resulting group is of order

$$
\begin{cases}2 \cdot 2 \cdot(2 s)^{2}=16 s^{2}, & \text { if } t \text { is odd, } \\ 2 \cdot(2 s)^{2}=8 s^{2}, & \text { if } t \text { is even. }\end{cases}
$$

Whatever $t$ is, we obtain a polyhedron with (planar) square vertex-figures and skew $2 s$-gonal faces, namely, one of type

$$
\left\{\frac{2 s}{t, s}, 4: \frac{4}{1,2}\right\} .
$$

The Petrial $\left\{\frac{4}{1,2}, 4: \frac{2 s}{t, s}\right\}$ is a toroid of type $\{4,4\}_{(s, s)}$ when $t$ is odd, but of type $\{4,4\}_{(s, 0)}$ if $t$ is even (note that the group order gives the type; we discuss the relationship between these toroids and those of the previous section in Section 11). Moreover, if $t$ is odd, then there is a polyhedron of type $\left\{\frac{4}{1,2}, \frac{2 s}{t}: \frac{4}{1,2}\right\}$ dual to the first (if $t$ is even, then the dual is not realizable in $\mathbb{E}^{4}$ ).

The two polyhedra in the family $\left\{3,3, \frac{3}{2}\right\}$ have the same group of order $2 \cdot 120=240$ (and the same 20 vertices) as $\{4,6 \mid 3\}$, and so are derived from the group [3,3,3] of the 4-simplex; we shall see why in Section 11. They form a Petrial pair, and are also interchanged by the operation $\kappa$. In the notation we introduced in Section 5, they are of type

$$
\left\{\frac{6}{1,3}, 6: \frac{6}{2,3}\right\}, \quad\left\{\frac{6}{2,3}, 6: \frac{6}{1,3}\right\} .
$$

Further, the first polyhedron is self-dual; the second has no dual in $\mathbb{E}^{4}$.

The remaining polyhedra in the class $(2,3,2)$ are all derived from the group $[3,4,3]$; the symmetry groups have order $2 \cdot 1152=2304$. Indeed, the hyperplane reflexions in the three diagrams are the same, and it is only the choice of outer normal vectors to them which gives rise to different diagrams; it is then the automorphisms which differ. In the last case $\{p, q, r\}=\left\{4, \frac{4}{3}, 3\right\}$, we have the whole family of six related by duality (interchange $R_{0}$ and $R_{2}$ ) and Petriality (replace $R_{0}$ by $R_{0} R_{2}$ ). For the other two (as we have said), $\frac{3}{2}$ cannot play the rôle of $q$.

We shall see in Section 11 that the polyhedra for which $q=4$ or $\frac{4}{3}$ have the same 144 vertices as $\{4,8 \mid 3\}$, namely, those of the (Minkowski) sum of $\{3,4,3\}$ and its dual (of the same size). When $q=3$ (recall that $q=\frac{3}{2}$ is not permitted), we have $192=2.96$ vertices; these are the mid-points of the edges of one copy of $\{3,4,3\}$ and those of its dual copy. 
Again, we list the polyhedra according to their diagrams:

$$
\begin{aligned}
& \left\{\frac{8}{1,4}, 8: \frac{6}{2,3}\right\}, \quad\left\{\frac{6}{2,3}, 8: \frac{8}{1,4}\right\}, \\
& \left\{\frac{8}{3,4}, \frac{8}{3}: \frac{6}{2,3}\right\}, \quad\left\{\frac{6}{2,3}, \frac{8}{3}: \frac{8}{3,4}\right\} \text {, } \\
& \left\{\frac{8}{1,4}, \frac{8}{3}: \frac{6}{1,3}\right\},\left\{\frac{6}{1,3}, \frac{8}{3}: \frac{8}{1,4}\right\},\left\{\frac{8}{3,4}, 6: \frac{8}{1,4}\right\},\left\{\frac{8}{1,4}, 6: \frac{8}{3,4}\right\},\left\{\frac{6}{1,3}, 8: \frac{8}{3,4}\right\},\left\{\frac{8}{3,4}, 8: \frac{6}{1,3}\right\} \text {. }
\end{aligned}
$$

In the first two lines, the pairs are mutual Petrials; the first of each pair is self-dual. In the last line, the polyhedra are related alternately by $\pi$ and $\delta$ (in a cycle). The operation $\kappa$ also permutes these polyhedra; it will preserve vertex-figures, but will interchange the pairs of marks $\left\{\frac{8}{1,4}, \frac{8}{3,4}\right\}$ and $\left\{\frac{6}{1,3}, \frac{6}{2,3}\right\}$ in both face and Petrie polygons. As an example,

$$
\left\{\frac{8}{1,4}, 8: \frac{6}{2,3}\right\} \stackrel{\kappa}{\longleftrightarrow}\left\{\frac{8}{3,4}, 8: \frac{6}{1,3}\right\} .
$$

Finally, $\varphi_{3}$ also links pairs of polyhedra whose vertex-figures are octagons. Namely, we first have

$$
\left\{\frac{8}{1,4}, 8: \frac{6}{2,3}\right\} \stackrel{\varphi_{3}}{\longleftrightarrow}\left\{\frac{8}{1,4}, \frac{8}{3}: \frac{6}{1,3}\right\}, \quad\left\{\frac{8}{3,4}, \frac{8}{3}: \frac{6}{2,3}\right\} \stackrel{\varphi_{3}}{\longleftrightarrow}\left\{\frac{8}{3,4}, 8: \frac{6}{1,3}\right\} .
$$

Of course, since $\pi$ and $\varphi_{3}$ commute, we can similarly link their Petrials:

$$
\left\{\frac{6}{2,3}, 8: \frac{8}{1,4}\right\} \stackrel{\varphi_{3}}{\longleftrightarrow}\left\{\frac{6}{1,3}, \frac{8}{3}: \frac{8}{1,4}\right\}, \quad\left\{\frac{6}{2,3}, \frac{8}{3}: \frac{8}{3,4}\right\} \stackrel{\varphi_{3}}{\longleftrightarrow}\left\{\frac{6}{1,3}, 8: \frac{8}{3,4}\right\} .
$$

\section{Quaternions}

As we said earlier, the symmetry groups with dimension vector $(2,2,2)$ are anomalous, in that there are no immediate relationships to reflexion groups. In fact, in some cases, no such relationship exists. Our treatment of this class will employ quaternions. In this section we give a brief outline of that part of the theory which we need; for further details, consult [10].

We recall that the quaternions consist of all $\mathbf{x}=\xi_{0}+\xi_{1} \mathbf{i}+\xi_{2} \mathbf{j}+\xi_{3} \mathbf{k}$, where $\xi_{j} \in \mathbb{R}$ for $i=0, \ldots, 3$, with an associative (but not commutative) multiplication induced by

$$
\mathbf{i}^{2}=\mathbf{j}^{2}=\mathbf{k}^{2}=\mathbf{i j k}=-1 .
$$

It is convenient to identify $\mathbf{x}$ with the vector $\left(\xi_{0}, \xi_{1}, \xi_{2}, \xi_{3}\right) \in \mathbb{E}^{4}$. The real part of $\mathbf{x}$ is $\Re(\mathbf{x})=\xi_{0}$, and its imaginary part is $\Im(\mathbf{x})=\xi_{1} \mathbf{i}+\xi_{2} \mathbf{j}+\xi_{3} \mathbf{k}$. The conjugate of $\mathbf{x}$ is $\overline{\mathbf{x}}=\xi_{0}-\xi_{1} \mathbf{i}-\xi_{2} \mathbf{j}-\xi_{3} \mathbf{k}$; then $\mathbf{x} \overline{\mathbf{x}}=\|\mathbf{x}\|^{2}$ (as a real vector). Thus, if $\mathbf{x} \neq 0$, then $\mathbf{x}$ is invertible. In particular, the set $\mathcal{Q}$ of unit quaternions (that is, with $\|\mathbf{x}\|=1$ ) forms a group; the inverse of $\mathbf{x} \in \mathcal{Q}$ is $\overline{\mathbf{x}}$.

Each element of the rotation group $\mathrm{SO}_{4}$ can be represented by a transformation of the form

$$
\mathbf{x} \mapsto \mathbf{x} \mathbf{g}(\mathbf{a}, \mathbf{b}):=\overline{\mathbf{a x}} \mathbf{b},
$$

where $\mathbf{a}, \mathbf{b} \in \mathcal{Q}$. In keeping with our usual conventions, mappings are thought of as acting on the right; thus it must be the inverse of a quaternion which provides an appropriate mapping when acting on the left. The group $\mathcal{G}$ of a polyhedron in the class $(2,2,2)$ is 
clearly a finite subgroup of $\mathrm{SO}_{4}$, and so admits a representation as a group consisting of such elements $\mathbf{g}(\mathbf{a}, \mathbf{b})$.

Before we go further, it is appropriate to comment on the rôle of conjugacy, which gives insights into the geometry of the mappings $\mathbf{g}(\cdot, \cdot)$ (compare [10] here). The leftacting quaternions a occurring in the group $\mathcal{G}$ clearly form a finite (left) subgroup $\mathcal{G}_{\mathrm{L}}$ of the whole group $\mathcal{Q}$ of unit quaternions. There is similarly a right subgroup $\mathcal{G}_{\mathrm{R}}$ consisting of the right-acting quaternions $\mathbf{b}$. Then it is easy to see that conjugating the whole group $\mathcal{G}$ leads to conjugating the left and right groups $\mathcal{G}_{\mathrm{L}}$ and $\mathcal{G}_{\mathrm{R}}$, and conversely. This freedom to take conjugates, which amounts to a free choice of suitable coordinates for the subgroups $\mathcal{G}_{\mathrm{L}}$ and $\mathcal{G}_{\mathrm{R}}$, will prove very useful.

In fact, $\mathcal{G}$ is a certain quotient of $\mathcal{G}_{\mathrm{L}} \times \mathcal{G}_{\mathrm{R}}$. If we define

$$
\mathcal{N}_{\mathrm{L}}:=\left\{\mathbf{a} \in \mathcal{G}_{\mathrm{L}} \mid \mathbf{g}(\mathbf{a}, 1) \in \mathcal{G}\right\}
$$

(that is, $\mathbf{x} \mapsto \overline{\mathbf{a}} \mathbf{x}$ is in $\mathcal{G}$ ), and $\mathcal{N}_{\mathrm{R}}$ similarly, then $\mathcal{N}_{\mathrm{L}}$ and $\mathcal{N}_{\mathrm{R}}$ are normal subgroups (of $\mathcal{G}_{\mathrm{L}}$ and $\mathcal{G}_{\mathrm{R}}$ ) such that $\mathcal{G}_{\mathrm{L}} / \mathcal{N}_{\mathrm{L}} \cong \mathcal{G}_{\mathrm{R}} / \mathcal{N}_{\mathrm{R}}$.

If $\mathbf{a}=\cos \vartheta+\sin \vartheta \mathbf{u}$ and $\mathbf{b}=\cos \varphi+\sin \varphi \mathbf{v}$, with $\mathbf{u}, \mathbf{v}$ pure imaginary unit quaternions, then $\mathbf{g}(\mathbf{a}, \mathbf{b})$ is (in general) a double rotation through angles $\pm \varphi \pm \vartheta$; thus it is a planar rotation (with a two-dimensional axis) just when $\varphi= \pm \vartheta$. It further follows that, for $\mathbf{g}(\mathbf{a}, \mathbf{b})$ to be a reflexion (that is, involutory), other than $\mathbf{x} \mapsto-\mathbf{x}$, both $\mathbf{a}$ and $\mathbf{b}$ must be pure imaginary.

The unit quaternion $\mathbf{a}=\cos \vartheta+\sin \vartheta \mathbf{u}$ induces an element of $\mathrm{SO}_{3}$, given by

$$
\mathbf{x} \mapsto \mathbf{x g}(\mathbf{a}, \mathbf{a})=\overline{\mathbf{a}} \mathbf{x a},
$$

where $\mathbf{x}$ is pure imaginary (thus we regard $\mathbb{E}^{3}$ as the subspace of pure imaginary quaternions); the kernel of this homomorphism from $\mathcal{Q}$ to $\mathrm{SO}_{3}$ is $\{ \pm 1\}$. This element is a rotation through the angle $2 \vartheta$ about the axis $\operatorname{lin}\{\mathbf{u}\}$ (or about $\operatorname{lin}\{1, \mathbf{u}\}$ if thought of as in $\mathbb{E}^{4}$ ). Thus the constituent groups $\mathcal{G}_{\mathrm{L}}$ and $\mathcal{G}_{\mathrm{R}}$ have quotients $G_{\mathrm{L}}$ and $G_{\mathrm{R}}$ in $\mathrm{SO}_{3}$, each (in the present case) of half the order; these will be generated by half-turns about lines in $\mathbb{E}^{3}$. Two such groups $\mathcal{G}, \mathcal{G}^{\prime}$ are conjugate in $\mathrm{SO}_{4}$ if and only if the corresponding pairs of rotation groups $G_{\mathrm{L}}, G_{\mathrm{L}}^{\prime}$ and $G_{\mathrm{R}}, G_{\mathrm{R}}^{\prime}$ are conjugate in $\mathrm{SO}_{3}$. However, the fact that each element of $\mathrm{SO}_{3}$ lifts to two elements of $\mathcal{Q}$ will cause some problems.

The standard scalar product in $\mathbb{E}^{4}$ is given by $\langle\mathbf{x}, \mathbf{y}\rangle=\Re(\overline{\mathbf{x}} \mathbf{y})$, and so we see that, if $\mathbf{a}, \mathbf{b} \in \mathcal{Q}$ are pure imaginary (so that $\mathbf{a}^{2}=\mathbf{b}^{2}=-1$ ), then $\mathbf{a b}$ is of the form $\cos \vartheta+\sin \vartheta \mathbf{u}$ for some pure imaginary $\mathbf{u} \in \mathcal{Q}$, where $\cos \vartheta=-\langle\mathbf{a}, \mathbf{b}\rangle$.

For future reference, we list the quaternion groups which play a rôle later. It must be emphasized, however, that the coordinates chosen here are, to a certain extent, arbitrary. Their elements are quaternions $v_{0}+v_{1} \mathbf{i}+v_{2} \mathbf{j}+v_{3} \mathbf{k}$, with the vectors $\left(v_{0}, v_{1}, v_{2}, v_{3}\right) \in \mathbb{E}^{4}$ taking the following values. First, the binary dihedral group $\mathcal{D}_{n}$ of order $4 n$ : all elements

$$
(\cos (k \pi / n), \sin (k \pi / n), 0,0), \quad(0,0, \cos (k \pi / n), \sin (k \pi / n)),
$$

for $k=0, \ldots, 2 n-1$. In fact, only the case $n$ even will concern us. Next, the binary octahedral group $\mathcal{O}$ of order 48: all permutations of

$$
( \pm 1,0,0,0), \quad \frac{1}{2}( \pm 1, \pm 1, \pm 1, \pm 1), \quad \frac{1}{2}( \pm \sqrt{2}, \pm \sqrt{2}, 0,0) .
$$


Note that these form the vertices of two dual regular 24-cells in $\mathbb{E}^{4}$. Last, the icosians or binary icosahedral group $\mathcal{I}$ of order 120: all even permutations of

$$
( \pm 1,0,0,0), \quad \frac{1}{2}( \pm 1, \pm 1, \pm 1, \pm 1), \quad \frac{1}{2}\left(0, \pm \tau, \pm \tau^{-1}, \pm 1\right),
$$

where $\tau=\frac{1}{2}(1+\sqrt{5})$ is the golden section. (The odd permutations would serve as well, and we shall not worry about which choice is made in any given context.) These form the vertices of a regular 600-cell in $\mathbb{E}^{4}$. Observe that the latter two groups have a common subgroup, the binary tetrahedral group $\mathcal{T}$, consisting of the first 24 elements of each (which form the vertices of a 24-cell). We shall see that the only groups which can occur as appropriate groups $G_{\mathrm{L}}$ or $G_{\mathrm{R}}$ are the dihedral groups $D_{2 n}$, the octahedral group $S_{4}$ or the icosahedral group $A_{5}$; the cyclic groups $C_{n}$, dihedral groups $D_{2 n+1}$ and tetrahedral group $A_{4}$ do not contain enough half-turns (and, in particular, are not generated by them).

Remark 9.1. A comment is in order here. An opposite orthogonal transformation of $\mathbb{E}^{4}$ is of the form

$$
\mathbf{x} \mapsto \overline{\mathbf{a}} \overline{\mathbf{x}} \mathbf{b},
$$

with $\mathbf{a}, \mathbf{b}$ as before. In a group $\mathcal{G}$ containing such transformations, the corresponding left and right groups $\mathcal{G}_{\mathrm{L}}$ and $\mathcal{G}_{\mathrm{R}}$ must be conjugate in the whole group of unit quaternions (again, see [10] for this). Thus one could also use quaternions to investigate the classes other than $(2,2,2)$; however, the methods which we have already described are more efficacious.

\section{Dimension Vector $(2,2,2)$}

Let $P$ be a regular polyhedron of Schläfli type $\{p, q\}$ (with $p, q$ generally fractional) in the class $(2,2,2)$, and let $\mathcal{G}=\left\langle R_{0}, R_{1}, R_{2}\right\rangle \leqslant \mathrm{SO}_{4}$ be its symmetry group, so that $\operatorname{dim} R_{j}=2$ for each $j$. As in Section 9, we regard $\mathcal{G}$ as a group of elements $\mathbf{g}(\mathbf{a}, \mathbf{b})$, with $\mathbf{a}, \mathbf{b} \in \mathcal{Q}$, the group of unit quaternions. Our approach will be to determine which rotation groups $G_{\mathrm{L}}, G_{\mathrm{R}} \leqslant \mathrm{SO}_{3}$ are involved, and how they lift to groups $\mathcal{G}_{\mathrm{L}}, \mathcal{G}_{\mathrm{R}} \leqslant \mathcal{Q}$.

We can therefore suppose that the generating reflexions are $R_{j}=\mathbf{g}\left(\mathbf{a}_{j}, \mathbf{b}_{j}\right)$ for $j=$ $0,1,2$, with each $\mathbf{a}_{j}, \mathbf{b}_{j} \in \mathcal{Q}$ pure imaginary (as we observed in Section 9). Interpreted as unit vectors in $\mathbb{E}^{3}$, we can think of $\mathbf{a}_{0}, \mathbf{a}_{1}, \mathbf{a}_{2}$ as outer normals to the cone generated by some Schwarz triangle on the unit sphere (see Section 6.8 of [4]). If this triangle is $\left(r_{1} q 2\right)$, it means that

$$
\left\langle\mathbf{a}_{0}, \mathbf{a}_{1}\right\rangle=-\cos \left(\pi / r_{1}\right), \quad\left\langle\mathbf{a}_{1}, \mathbf{a}_{2}\right\rangle=-\cos (\pi / q), \quad\left\langle\mathbf{a}_{0}, \mathbf{a}_{2}\right\rangle=0 .
$$

Similarly, $\mathbf{b}_{0}, \mathbf{b}_{1}, \mathbf{b}_{2}$ give a Schwarz triangle ( $r_{2} q 2$ ). To some extent, this already fixes the possible liftings into quaternions; however, there is still ambiguity as to the choice of signs of (say) the $\mathbf{a}_{j}$, bearing in mind that $\mathbf{g}(-\mathbf{a},-\mathbf{b})=\mathbf{g}(\mathbf{a}, \mathbf{b})$.

Since $R_{1} R_{2}$ is an ordinary rotation through $2 \pi / q$ (and we can take $q>2$ without loss of generality), we see that we must have

$$
\left\langle\mathbf{a}_{1}, \mathbf{a}_{2}\right\rangle=\left\langle\mathbf{b}_{1}, \mathbf{b}_{2}\right\rangle= \pm \cos (\pi / q) .
$$


By changing signs of both $\mathbf{a}_{1}$ and $\mathbf{b}_{1}$ if necessary, we can assume that the sign is negative, fitting in with the convention of Section 9. Further, we have $r_{1}, r_{2}>1$ such that

$$
\left\langle\mathbf{a}_{0}, \mathbf{a}_{1}\right\rangle=-\cos \left(\pi / r_{1}\right), \quad\left\langle\mathbf{b}_{0}, \mathbf{b}_{1}\right\rangle=-\cos \left(\pi / r_{2}\right) ;
$$

we shall see that it is necessary to allow $r_{j} \leqslant 2$.

We first look at just one of the groups $\mathcal{G}_{\mathrm{L}}$ and $\mathcal{G}_{\mathrm{R}}$, say, the former. Here, we make the choice of coordinates

$$
\begin{aligned}
& \mathbf{a}_{0}=\mathbf{i}, \\
& \mathbf{a}_{1}=\alpha_{1} \mathbf{i}+\alpha_{2} \mathbf{j}+\alpha_{3} \mathbf{k}, \\
& \mathbf{a}_{2}=\mathbf{j} .
\end{aligned}
$$

We therefore have

$$
\alpha_{1}=-\cos \left(\pi / r_{1}\right), \quad \alpha_{2}=-\cos (\pi / q)
$$

Since

$$
\mathbf{a}_{0} \mathbf{a}_{2}=\mathbf{i j}=\mathbf{k}
$$

it follows that

$$
\alpha_{3}=-\cos \left(\pi / h_{1}\right),
$$

where we regard $G_{\mathrm{L}}$ as the group of a (possibly degenerate) polyhedron $\left\{r_{1}, q: h_{1}\right\}$, with the notation $h_{1}$ indicating its Petrie type (this may perhaps be better thought of as a polyhedron lying in the real projective plane). Note that

$$
\cos ^{2}\left(\pi / r_{1}\right)+\cos ^{2}(\pi / q)+\cos ^{2}\left(\pi / h_{1}\right)=\alpha_{1}^{2}+\alpha_{2}^{2}+\alpha_{3}^{2}=1 ;
$$

this gives a different way of looking at Coxeter's formula [4, 2.33], which is easily deducible from it.

We gain even more information (enough to distinguish among various possibilities) if we look further into the geometry. Recalling the free choice of coordinates we have for our generating quaternions $\mathbf{a}_{i}$ and $\mathbf{b}_{j}$, we now pick them to be

$$
\begin{aligned}
& \mathbf{a}_{0}=\alpha_{1} \mathbf{i}+\alpha_{2} \mathbf{j}, \quad \mathbf{b}_{0}=\beta_{1} \mathbf{i}+\beta_{2} \mathbf{j}, \\
& \mathbf{a}_{1}=\mathbf{b}_{1}=\sin (\pi / q) \mathbf{j}-\cos (\pi / q) \mathbf{k}, \\
& \mathbf{a}_{2}=\mathbf{b}_{2}=\mathbf{k} .
\end{aligned}
$$

(We remark that these coordinates are only chosen to elucidate the geometry; they will not usually be best in other circumstances.) In view of our previous discussion, we have

$$
\alpha_{2} \sin (\pi / q)=-\cos \left(\pi / r_{1}\right) \quad \Longrightarrow \quad \alpha_{2}=-\frac{\cos \left(\pi / r_{1}\right)}{\sin (\pi / q)},
$$

and similarly for $\beta_{2}$. (Recall that we only insist that $r_{1}>1$, so that $\cos \left(\pi / r_{1}\right)<0$ is permitted.) Finally, applying the Petrie operation, and so replacing $\mathbf{a}_{0}$ by

$$
\mathbf{a}_{0} \mathbf{a}_{2}=\left(\alpha_{1} \mathbf{i}+\alpha_{2} \mathbf{j}\right) \mathbf{k}=\alpha_{2} \mathbf{i}-\alpha_{1} \mathbf{j},
$$


we see that

$$
-\alpha_{1} \sin (\pi / q)=-\cos \left(\pi / h_{1}\right) \quad \Longrightarrow \quad \alpha_{1}=\frac{\cos \left(\pi / h_{1}\right)}{\sin (\pi / q)},
$$

and similarly for $\beta_{1}$.

At this stage, we begin to see the rôle that different liftings of the groups $G_{\mathrm{L}}$ and $G_{\mathrm{R}}$ can play. Since we may transfer a change of sign freely between $\mathbf{a}_{j}$ and $\mathbf{b}_{j}$, we need only impose such sign changes on the $\mathbf{a}_{j}$ (in practice, we do not always do this, particularly when we wish to emphasize some symmetry). Moreover, we must ensure that $\left\langle\mathbf{a}_{1}, \mathbf{a}_{2}\right\rangle=\left\langle\mathbf{b}_{1}, \mathbf{b}_{2}\right\rangle=-\cos (\pi / q)$, and so $\mathbf{a}_{1}$ and $\mathbf{a}_{2}$ must change sign together. Thus our freedom of choice is to change the sign of $\mathbf{a}_{0}$, change signs of both $\mathbf{a}_{1}$ and $\mathbf{a}_{2}$, or change all three signs. The first is $\kappa$, and replaces $r_{1}$ and $h_{1}$ by $r_{1}^{\prime}$ and $h_{1}^{\prime}$, given by

$$
\frac{1}{r_{1}}+\frac{1}{r_{1}^{\prime}}=1, \quad \frac{1}{h_{1}}+\frac{1}{h_{1}^{\prime}}=1
$$

The second replaces $r_{1}$ by $r_{1}^{\prime}$, while the third replaces $h_{1}$ by $h_{1}^{\prime}$. Potentially, then, this gives four distinct liftings, giving rise to four different polyhedra. Bearing in mind what we said about congruency of these groups in Section 9, we can see that equivalence of the sets of vectors $\left(\mathbf{a}_{0}, \mathbf{a}_{1}, \mathbf{a}_{2}\right)$ and $\left(\mathbf{b}_{0}, \mathbf{b}_{1}, \mathbf{b}_{2}\right)$ under $\mathrm{SO}_{3}$ is what matters, subject to possible exchanges of sign of an $\mathbf{a}_{j}$ and corresponding $\mathbf{b}_{j}$. It should be noticed that changing the signs of all three $\mathbf{a}_{j}$ (which is the easiest way of changing relative orientation) will, in general, not yield the same polyhedron (if it exists), because $h_{1}$ is replaced by $h_{1}^{\prime}$ while $r_{1}$ is preserved.

Our notation keeps track of these sign changes. The triple $r_{j}, q, h_{j}$ is associated with the regular projective polyhedron $\left\{r_{j}, q: h_{j}\right\}$ (which indicates the type of the polyhedron and its Petrial), with the understanding that replacing $r_{j}$ by $r_{j}^{\prime}$ or $h_{j}$ by $h_{j}^{\prime}$ gives the same set of generators of a finite subgroup of $\mathrm{SO}_{3}$, but that different choices may give different liftings of the combined pair of groups.

From the discussion of Section 9, we already know the type of face of our polyhedron. It is of the form $\left\{p_{1}\right\} \#\left\{p_{2}\right\}$, with the $p_{j}$ given by

$$
\frac{1}{p_{j}}= \pm \frac{1}{2}\left(\frac{1}{r_{1}} \pm \frac{1}{r_{2}}\right)
$$

for some choice of signs (different inside the bracket); here, we replace $r_{i}$ by $r_{i}^{\prime}$ for both $i$ if necessary, to ensure that suitable signs can be chosen so that $p_{j}>2$ for $j=1,2$ (it is easy to see that this is possible). This choice, by the way, may result in changing signs of some $\mathbf{b}_{j}$ (as well as, of course, the corresponding $\mathbf{a}_{j}$ ). As in Section 5, it is convenient to write the face, instead, as

$$
\left\{\frac{p}{d_{1}, d_{2}}\right\}, \quad \text { with } \quad p_{j}=\frac{p}{d_{j}}
$$

(when reduced to lowest terms) for $j=1,2$. Thus we have $1 \leqslant d_{1}<d_{2}<\frac{1}{2} p$; the faces are helices, so that $d_{2}=\frac{1}{2} p$ is not allowed. 
Observe that, with the correct choice of signs above, $r_{1}$ and $r_{2}$ can be recovered from

$$
\frac{1}{r_{j}}=\frac{d_{2} \pm d_{1}}{p}
$$

with reduction of the fractions to their lowest terms. These are the $r_{1}$ and $r_{2}$ which we usually employ in the notation for the polyhedra.

The type of the Petrie polygon is given in exactly the same way, with $h_{1}, h_{2}$ instead of $r_{1}, r_{2}$. Using the conventions just introduced, we denote the resulting polyhedron (when it exists) by

$$
\left\{r_{1}, q: h_{1}\right\} \bowtie\left\{r_{2}, q: h_{2}\right\} .
$$

Our present choice of coordinates gives 1 as an initial vertex of the polyhedron (assuming that it exists); then the adjacent vertex $1 R_{0}$ is

$$
\overline{\mathbf{a}_{0}} 1 \mathbf{b}_{0}=-\mathbf{a}_{0} \mathbf{b}_{0}=-\left(\alpha_{1} \mathbf{i}+\alpha_{2} \mathbf{j}\right)\left(\beta_{1} \mathbf{i}+\beta_{2} \mathbf{j}\right)=\left(\alpha_{1} \beta_{1}+\alpha_{2} \beta_{2}\right)+\left(\alpha_{2} \beta_{1}-\alpha_{1} \beta_{2}\right) \mathbf{k},
$$

so that, if the edge-length is $2 \lambda$, then

$$
\begin{aligned}
\lambda^{2} & =\frac{1}{4}\left(\left(1-\left(\alpha_{1} \beta_{1}+\alpha_{2} \beta_{2}\right)\right)^{2}+\left(\alpha_{2} \beta_{1}-\alpha_{1} \beta_{2}\right)^{2}\right) \\
& =\frac{1}{2}\left(1-\alpha_{1} \beta_{1}-\alpha_{2} \beta_{2}\right) \\
& =\frac{1}{2}\left(1-\frac{\cos \left(\pi / h_{1}\right) \cos \left(\pi / h_{2}\right)+\cos \left(\pi / r_{1}\right) \cos \left(\pi / r_{2}\right)}{\sin ^{2}(\pi / q)}\right),
\end{aligned}
$$

after a little calculation (note that $\alpha_{1}^{2}+\alpha_{2}^{2}=1$, and so on).

Remark 10.1. Because the symmetries of a polyhedron in this class consist of rotations only, such polyhedra occur in enantiomorphic pairs, where the 2-faces are always left or always right helices. (The situation is exactly analogous to that of the regular apeirohedra in $\mathbb{E}^{3}$ whose symmetry groups have dimension vector $(1,1,1)$; compare Remark 4.1. See [21] or Section 7E of [22] for further details about these apeirohedra.) Any opposite isometry- $\mathrm{in}_{4} \backslash \mathrm{SO}_{4}$-interchanges the enantiomorphism classes.

Remark 10.2. The whole symmetry groups $[3,4]$ and $[3,5]$ of the octahedron and icosahedron are not rotation groups. Notice, by the way, that this accords with the wellknown facts that [3, 4] $\cong S_{4} \times C_{2}$ and [3,5] $\cong A_{5} \times C_{2}$, with $C_{2}$ here generated by the reflexion in $\{o\}$ (the central inversion).

One problem which we encounter here is that the corresponding polyhedron may degenerate, although there are pointers to when degeneracy occurs. As a specific example, ignoring the rôle of the $h_{j}$ for the moment, consider the case $r_{1}=3, r_{2}=\frac{5}{2}$ and $q=5$, when we should obtain a polyhedron

$$
\{3,5\} \bowtie\left\{\frac{5}{2}, 5\right\}=\left\{\frac{30}{1,11}, 5\right\},
$$

with the latter symbol indicating its type. If we replace $\frac{5}{2}$ by $\frac{5}{3}$ (or 3 by $\frac{3}{2}$ ), giving the other choice of lifting for $R_{0}$, then we should obtain type

$$
\{3,5\} \bowtie\left\{\frac{5}{3}, 5\right\}=\left\{\frac{15}{2,7}, 5\right\} .
$$


(Up to enantiomorphism, these two choices can be Petrials of each other.) Now reference to [10] shows that the only possible group these polyhedra can have is the rotation group $[3,3,5]^{+}$of the regular 600 -cell $\{3,3,5\}$, of order 7200 . (There are limited possibilities which ensure that $\mathcal{G}_{\mathrm{L}} / \mathcal{N}_{\mathrm{L}} \cong \mathcal{G}_{\mathrm{R}} / \mathcal{N}_{\mathrm{R}}$, because the 120 icosians $\mathcal{I}$ of (9.4) form a double cover of the simple group $A_{5}$.) Thus the polyhedra themselves should have 7200/10= 720 vertices. However, if we choose (for the first polyhedron) $\mathcal{G}_{\mathrm{L}}=\left\langle\mathbf{a}_{0}, \mathbf{a}_{1}, \mathbf{a}_{2}\right\rangle$ and $\mathcal{G}_{\mathrm{R}}=\left\langle\mathbf{b}_{0}, \mathbf{b}_{1}, \mathbf{b}_{2}\right\rangle$, with generators

$$
\begin{aligned}
& \mathbf{a}_{0}=\mathbf{k}, \quad \mathbf{b}_{0}=\mathbf{j}, \\
& \mathbf{a}_{1}=\mathbf{b}_{1}=-\frac{1}{2}\left(\tau \mathbf{i}+\tau^{-1} \mathbf{j}+\mathbf{k}\right), \\
& \mathbf{a}_{2}=\mathbf{b}_{2}=\mathbf{i},
\end{aligned}
$$

where $\tau=\frac{1}{2}(1+\sqrt{5})$, in which case (up to scaling) 1 is the initial vertex, it is obvious that the vertices obtained form a subset of $\mathcal{I}$, that is, the vertex set of $\{3,3,5\}$; in fact, we get all of $\mathcal{I}$. It follows that the 720 putative vertices of the two polyhedra must coincide in sixes at the vertices of the 600-cell, and so the polyhedra degenerate. (We shall return to this example later, and see that it can be redeemed by - for example — changing signs of all the $\mathbf{b}_{j}$. In view of this degeneracy, though, it was unfortunate that we chose it as an illustrative example in [23].)

We must thus look at the possible component rotation subgroups of $\mathrm{SO}_{3}$ systematically. We first list those groups which contribute to the enumeration; these are really groups which act on the corresponding projective space and, as we have seen, we can specify them by (rational) triples $\{p, q, r\}$ with

$$
\cos ^{2}(\pi / p)+\cos ^{2}(\pi / q)+\cos ^{2}(\pi / r)=1 .
$$

This is Gordan's equation. Here, $p, q, r$ stand indiscriminately for the type of the face, vertex-figure and Petrie polygon. In lifting them, we can replace (for example) $p$ by $p^{\prime}$, except that the mark $q$, say, for the vertex-figure always satisfies $q>2$. In the listing we also need to define $q^{\prime \prime}$ by

$$
\frac{1}{q}+\frac{1}{q^{\prime \prime}}=\frac{1}{2}
$$

We then have

$$
\left\{2, q, q^{\prime \prime}\right\}(q>2 \text { arbitrary }), \quad\{3,3,4\}, \quad\left\{3,5, \frac{5}{2}\right\} .
$$

Remark 10.3. We have not used the fact that these triples are (effectively) the only rational solutions of Gordan's equation (10.2), because our listing is derived from the rotation groups.

For the binary dihedral group, let $q=s / t>2$. The half-turns $R_{1}^{\prime}$ and $R_{2}^{\prime}$ in $\mathbb{E}^{3}$ corresponding to $R_{1}$ and $R_{2}$ are about axes subtending the angle $\pi(1-1 / q)$, ensuring that $R_{1} R_{2}$ is a single rotation through $\pi / q$; we can take the corresponding quaternions to be

$$
\begin{aligned}
& \mathbf{a}_{1}=\mathbf{b}_{1}=-(\cos (\pi / q) \mathbf{j}+\sin (\pi / q) \mathbf{k}), \\
& \mathbf{a}_{2}=\mathbf{b}_{2}=\mathbf{j} .
\end{aligned}
$$


The half-turn $R_{0}^{\prime}$ corresponding to $R_{0}$ must have an axis orthogonal to that of $R_{2}^{\prime}$; it can be in the plane of $R_{1}^{\prime}$ and $R_{2}^{\prime}$ (regarded as lines) or orthogonal to it. The different choices for the corresponding quaternions are thus

$$
\mathbf{a}_{0}= \pm \mathbf{i}, \quad \mathbf{b}_{0}= \pm \mathbf{k}
$$

We list them like this, but note that $a_{0}=\mathbf{k}$ (and so on) is equally possible. This gives the triple $\left\{2, q, q^{\prime \prime}\right\}$.

Which dihedral group $\left\langle R_{0}^{\prime}, R_{1}^{\prime}, R_{2}^{\prime}\right\rangle$ results depends on whether or not $s$ is even. In fact, we obtain

$$
\begin{cases}D_{s}, & \text { if } s \text { is even, } \\ D_{2 s}, & \text { if } s \text { is odd. }\end{cases}
$$

To see why, note that $\left\langle R_{1}^{\prime}, R_{2}^{\prime}\right\rangle \cong D_{s}$, and $R_{0}^{\prime} \in\left\langle R_{1}^{\prime}, R_{2}^{\prime}\right\rangle$ if $s$ is even, while $R_{0}^{\prime} \notin\left\langle R_{1}^{\prime}, R_{2}^{\prime}\right\rangle$ if $s$ is odd. Thus the quaternion group is $\mathcal{D}_{s}$ if $s$ is even, but $\mathcal{D}_{2 s}$ if $s$ is odd (see (9.2)).

After only a little work, we find that $\left\{2, q: q^{\prime \prime}\right\} \bowtie\left\{q^{\prime \prime}, q: 2\right\}$ has $2 s$ vertices

$$
\cos \frac{2 k \pi}{q}+\sin \frac{2 k \pi}{q} \mathbf{i}, \quad \cos \frac{2 k \pi}{q} \mathbf{j}+\sin \frac{2 k \pi}{q} \mathbf{k},
$$

for $k=0, \ldots, s-1$, where $q=s / t$ (in lowest terms) as before. In our abbreviated notation,

$$
\left\{2, q: q^{\prime \prime}\right\} \bowtie\left\{q^{\prime \prime}, q: 2\right\}=\left\{\frac{2 s}{t, s-t}, q: \frac{2 s}{t, s-t}\right\} ;
$$

the faces are therefore $2 s$-gons, so that the polyhedron is (combinatorially) flat, meaning that every vertex belongs to every face (see Sections $4 \mathrm{E}$ and $4 \mathrm{~F}$ of [22]). Moreover, it is self-Petrie (strictly, its Petrial is enantiomorphic), and is taken into a congruent copy by $\kappa$. Our four potential possibilities here reduce to one; note that changing signs of $\mathbf{a}_{1}$ and $\mathbf{a}_{2}$ leads to a directly congruent set in $\mathbb{E}^{3}$, as does changing signs of all three $\mathbf{b}_{j}$ for the second set. The symmetry group has order $4 s^{2}$. It is worth remarking why this order does not depend on $s$ being even or odd; if $s$ is even, the normal subgroups $\mathcal{N}_{\mathrm{L}}$ and $\mathcal{N}_{\mathrm{R}}$ are both $\mathcal{D}_{s / 2}$, while if $s$ is odd they are $\mathcal{C}_{s}$, a cyclic group of order $s$. (It should be remarked that this case was omitted in the brief sketch in [23].)

Next, we consider the case when one of the component quaternion groups, $\mathcal{G}_{\mathrm{L}}$ say, is binary dihedral, while $\mathcal{G}_{\mathrm{R}}$ is not. Then $q=3,4,5$ or $\frac{5}{2}$, and the other component $\mathcal{G}_{\mathrm{R}}$ is $\mathcal{O}$ (see $(9.3)$ ) or $\mathcal{I}$. Here, different liftings will generally give different polyhedra. For example,

$$
\begin{aligned}
& \{2,3: 6\} \bowtie\left\{5,3: \frac{5}{2}\right\}=\left\{\frac{20}{3,7}, 3: \frac{60}{7,17}\right\}, \\
& \{2,3: 6\} \bowtie\left\{5,3: \frac{5}{3}\right\}=\left\{\frac{20}{3,7}, 3: \frac{60}{13,23}\right\} .
\end{aligned}
$$

However, we get nothing new if we change 5 to $\frac{5}{4}$; the variation is in the Petrie polygon alone. Of course, if we take the Petrial, the opposite is true.

The enumeration is straightforward; we list Petrial pairs together. For $q=3$, we have two pairs with $\mathcal{G}_{\mathrm{R}}=\mathcal{O}$, and group order $24 \cdot 48 / 2=576$, and four pairs with $\mathcal{G}_{\mathrm{R}}=\mathcal{I}$, and group order $24 \cdot 120 / 2=1440$. For $q=4,5$ or $\frac{5}{2}$, we have two pairs each; for the 
first, $\mathcal{G}_{\mathrm{R}}=\mathcal{O}$ and the group has order $16 \cdot 48 / 2=384$, and for the other two $\mathcal{G}_{\mathrm{R}}=\mathcal{I}$ and the group order is $40 \cdot 120 / 2=2400$. In total, we thus obtain 24 polyhedra of this kind.

For the remainder, the component groups $\mathcal{G}_{\mathrm{L}}$ and $\mathcal{G}_{\mathrm{R}}$ are binary octahedral or binary icosahedral. The case where we have one of each-say $\mathcal{G}_{\mathrm{L}}=\mathcal{O}$ and $\mathcal{G}_{\mathrm{R}}=\mathcal{I}$-is the easiest, and so we treat that first. Here, we have $q=3$; the group order is $48 \cdot 120 / 2=$ 2880 , and so the polyhedra all have $2880 / 6=480$ vertices (note that these comprise four left cosets of $\mathcal{I}$ or ten right cosets of $\mathcal{O}$ ). For example, the polyhedra derived from $\{4,3: 3\}$ and $\left\{5,3: \frac{5}{2}\right\}$, with possible replacement of 5 by $\frac{5}{4}$ or $\frac{5}{2}$ by $\frac{5}{3}$, have edge-lengths $2 \lambda$ (and unit circumradius) given by

$$
\begin{aligned}
\lambda^{2} & =\frac{1}{2}\left(1-\frac{ \pm 1 / \sqrt{2} \cdot \frac{1}{2} \tau \pm \frac{1}{2} \cdot \frac{1}{2} \tau^{-1}}{\frac{3}{4}}\right) \\
& =\frac{1}{6}\left(3 \mp \tau \sqrt{2} \mp \tau^{-1}\right) .
\end{aligned}
$$

Thus the different liftings-corresponding to the choices of signs—give four distinct polyhedra. It is extremely tedious to verify that these polyhedra actually exist, because no convenient coordinates are available. In all, we obtain four Petrial pairs here, and another four from interchanging 5 and $\frac{5}{2}$ (and thus $\tau$ by $\tau^{-1}$ ). This gives 16 polyhedra in all, which we shall not list individually.

Remark 10.4. The representation of finite subgroups of $\mathrm{SO}_{4}$ by left and right quaternion groups has certain implications. For instance, the vertices of any polytope whose symmetry group is $[3,3,5]^{+}$or $[3,3,5]$ fall into subsets, each forming a copy of the vertex-set of $\{3,3,5\}$; its dual $\{5,3,3\}$ is a particular example.

Finally, we take the two component groups to be the same. We begin with the binary octahedral group. Here, we find that some liftings give degenerate polyhedra. In order to see why some choices lead to degeneracy, we must specify suitable generating reflexions. We thus first take

$$
\begin{aligned}
& \mathbf{a}_{0}=\frac{1}{\sqrt{2}}(\mathbf{j}+\mathbf{k}), \quad \mathbf{b}_{0}=-\mathbf{i}, \\
& \mathbf{a}_{1}=\mathbf{b}_{1}=\frac{1}{\sqrt{2}}(\mathbf{i}-\mathbf{j}), \\
& \mathbf{a}_{2}=\mathbf{b}_{2}=\frac{1}{\sqrt{2}}(\mathbf{j}-\mathbf{k}) .
\end{aligned}
$$

The Petrie operation $\pi$ replaces $\mathbf{a}_{0}$ and $\mathbf{b}_{0}$ by

$$
\mathbf{a}_{0}^{\prime}=\mathbf{a}_{0} \mathbf{a}_{2}=-\mathbf{i}, \quad \mathbf{b}_{0}^{\prime}=\mathbf{b}_{0} \mathbf{b}_{2}=-\frac{1}{\sqrt{2}}(\mathbf{j}+\mathbf{k}),
$$

so that the (putative) polyhedron would be

$$
\{3,3: 4\} \bowtie\left\{4,3: \frac{3}{2}\right\} .
$$


Note that this would not be self-Petrie. Since the initial vertex can clearly be taken to be 1 (as in our earlier general discussion), we see that the vertex-set is a subset of $\mathcal{O}$ (regarded as a set of points in $\mathbb{E}^{4}$ ); it is not hard to see that we obtain all these 48 points. However, the group has order $48^{2} / 2=1152$ (it is not the group $[3,4,3]$ of the 24-cell but, instead, the extension of its rotation subgroup $[3,4,3]^{+}$by an involutory outer automorphism), and so we should expect (if the polyhedron were non-degenerate) $1152 / 6=192$ vertices. It follows that the vertices actually collapse in sets of four.

The other non-self-Petrie cases degenerate in the same way. It is not immediately clear that the self-Petrie liftings do not degenerate, but we shall see why (by means of a direct construction) in Section 11. Given that they exist, we then obtain two polyhedra:

$$
\begin{gathered}
\{3,3: 4\} \bowtie\{4,3: 3\}=\left\{\frac{24}{1,7}, 3: \frac{24}{1,7}\right\}, \\
\left\{\frac{3}{2}, 3: 4\right\} \bowtie\left\{4,3: \frac{3}{2}\right\}=\left\{\frac{24}{5,11}, 3: \frac{24}{5,11}\right\} .
\end{gathered}
$$

Observe that these two polyhedra are related by $\kappa$.

We now move on to the binary icosahedral group $\mathcal{I}$. We first choose

$$
\begin{aligned}
& \mathbf{a}_{0}=\mathbf{i}, \quad \mathbf{b}_{0}=\mathbf{j}, \\
& \mathbf{a}_{1}=\mathbf{b}_{1}=-\frac{1}{2}\left(\tau \mathbf{i}-\tau^{-1} \mathbf{j}+\mathbf{k}\right), \\
& \mathbf{a}_{2}=\mathbf{b}_{2}=\mathbf{k} .
\end{aligned}
$$

Ignoring the Petrie polygon, we obtain the type $\{5,3\} \bowtie\left\{\frac{5}{3}, 3\right\}=\left\{\frac{5}{1,2}, 3\right\}$. If we apply the Petrie operation $\pi$, we replace $\mathbf{a}_{0}$ and $\mathbf{b}_{0}$ by

$$
\mathbf{a}_{0}^{\prime}=\mathbf{i k}=-\mathbf{j}, \quad \mathbf{b}_{0}^{\prime}=\mathbf{j k}=\mathbf{i},
$$

and the type is $\left\{\frac{5}{2}, 3\right\} \bowtie\{5,3\}=\left\{\frac{10}{1,3}, 3\right\}$. In other words, what we actually have for the first is

$$
\left\{5,3: \frac{5}{2}\right\} \bowtie\left\{\frac{5}{3}, 3: 5\right\}=\left\{\frac{5}{1,2}, 3: \frac{10}{1,3}\right\} .
$$

This identifies it as the faithful realization in $\mathbb{E}^{4}$ of the dodecahedron $\{5,3\}$, and the second polyhedron as that of its Petrial $\{10,3\}_{5}$. (See [15] or pp. 137-138 of [22] for more details about the realization space of $\{5,3\}$.) The group order is 120 , and the two polyhedra are related by $\kappa$ as well as $\pi$.

If, however, we change the sign of each $\mathbf{b}_{j}$ (rather than the $\mathbf{a}_{j}$ for convenience here), then the Petrie operation $\pi$ yields

$$
\mathbf{a}_{0}^{\prime}=\mathbf{i k}=-\mathbf{j}, \quad \mathbf{b}_{0}^{\prime}=\mathbf{j}(-\mathbf{k})=-\mathbf{i}
$$

as before, but the type is now

$$
\left\{5,3: \frac{5}{3}\right\} \bowtie\left\{\frac{5}{3}, 3: 5\right\}=\left\{\frac{5}{1,2}, 3: \frac{5}{1,2}\right\} .
$$

In this case we therefore have the faithful realization in $\mathbb{E}^{4}$ of the self-Petrie hemidodecahedron $\{5,3\}_{5}$ (in view of our previous remark, the two Petrials are actually enantiomorphic). Finally, changing the sign of $\mathbf{b}_{0}$ again, we now get

$$
\left\{5,3: \frac{5}{2}\right\} \bowtie\left\{\frac{5}{2}, 3: 5\right\}=\left\{\frac{10}{1,3}, 3: \frac{10}{1,3}\right\},
$$


for the polyhedron and its Petrial, which identifies it as the polyhedron of [16] whose graph is the generalized Petersen graph $G(10,3)$ of [12] (these are of type $\{p, 3\}$ with $2 p$ vertices-see also below). The first of the two polyhedra has group order 60 , and the second 120 . The two polyhedra are related by $\kappa$, which here changes the group order.

Remark 10.5. This case is in contrast to that of the binary octahedral group, in that all four polyhedra exist (that is, are non-degenerate). Note, however, that the group orders are much smaller than the $120 \cdot 120 / 2=7200$ that can occur when the component groups are both $\mathcal{I}$.

The remaining examples (in the binary icosahedral family) display exactly the same behaviour as those which arise when $\mathcal{G}_{\mathrm{L}}$ and $\mathcal{G}_{\mathrm{R}}$ are the binary octahedral group. In other words, when the putative polyhedron is self-Petrie, then it exists; if it is not, then it degenerates (once again, we make a forward reference to Section 11). For example, the degenerate case which we looked at earlier turns out to be

$$
\left\{3,5: \frac{5}{3}\right\} \bowtie\left\{\frac{5}{2}, 5: 3\right\}=\left\{\frac{30}{1,11}, 5: \frac{15}{2,7}\right\} .
$$

There are just two edge-lengths $2 \lambda$ of the polyhedra in the non-degenerate family, given by

$$
\lambda^{2}=\frac{1}{2}(1 \pm 2 / \sqrt{5}) .
$$

However, the effect of the facetting operation $\varphi_{2}$ should be noticed; this ties the two families of two (related by $\kappa$-they are already self-Petrie) into a single family of four. We then have

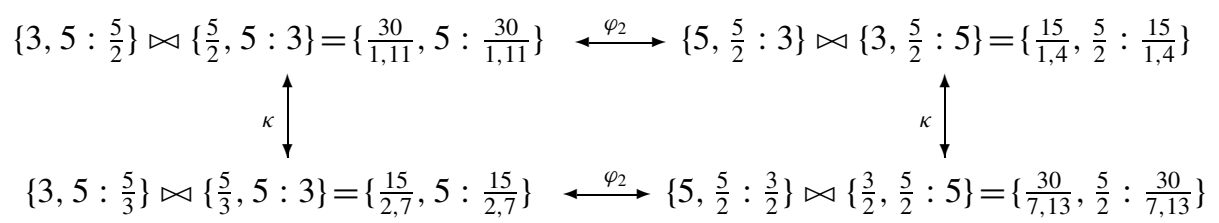

\section{Further Connexions}

In this final section we discuss some further connexions among the various classes which we have considered previously. Some of the operations which we introduced in Section 3 automatically lead from one polyhedron to another in the same class; thus, what we shall do in this section is look at the remaining possibilities. We have already dealt with $\delta$ and $\kappa$ in the general discussions. We have also observed the effect of $\pi$ in the way that we have grouped the classes. The only facetting operations $\varphi_{k}$ which play a rôle are $\varphi_{2}$ for polyhedra with pentagonal vertex-figures and $\varphi_{3}$ for polyhedra with octagonal vertexfigures, and we have already listed their effects, because they preserve the respective classes. Finally, then, we are left with halving $\eta$ and skewing $\sigma$.

For $\eta$, if the original dimension vector is $\left(d_{0}, d_{1}, d_{2}\right)$, then the new one is $\left(d_{1}, d_{2}, d_{1}\right)$; in our cases, we have $(3,2,3) \mapsto(2,3,2)$ or $(2,3,2) \mapsto(3,2,3)$. Note, however, that none of the polyhedra in the other classes related to $(3,2,3)$ permit the application of $\eta$, and that only the toroidal polyhedra in class $(2,3,2)$ permit it. 
It is straightforward to keep track of the generating (hyperplane) reflexions of the original diagrams of Figs. 1 and 2, to show that

$$
\mathcal{D}_{1}(2, q ; r) \stackrel{\eta}{\longmapsto} \mathcal{D}_{2}\left(q, q, \frac{r}{2}\right), \quad \mathcal{D}_{2}(2, q, r) \stackrel{\eta}{\longmapsto} \mathcal{D}_{1}(q, q ; r) ;
$$

that is, corresponding to the diagram $\mathcal{D}_{1}(2, q ; r)$ in class $(3,2,3)$ with $p=2$ (and so with the relevant branch of the diagram missing) is the diagram $\mathcal{D}_{2}\left(q, q, \frac{r}{2}\right)$ in class $(2,3,2)$ with $p=q$ and $r$ replaced by $\frac{r}{2}$, and similarly for $\mathcal{D}_{2}(2, q, r)$. We look at the individual cases.

We begin with the toroidal polyhedra and their relatives. For those of Schläfli type $\{4,4\}$, we have

$$
\left\{4, \frac{4}{1,2} \mid \frac{s}{t}\right\} \stackrel{\eta}{\longmapsto}\left\{\frac{4}{1,2}, 4: \frac{2 s}{t, s}\right\}, \quad\left\{\frac{4}{1,2}, 4: \frac{2 s}{t, s}\right\} \stackrel{\eta}{\longmapsto}\left\{4, \frac{4}{1,2} \mid \frac{s}{t}\right\} .
$$

Note that if $t$ is even, then $\eta$ does not halve the group order in the latter case. If $r=s / t$ with $t$ odd, then the dual of its Petrial is

$$
\left\{\frac{4}{1,2}, \frac{2 s}{t}: \frac{4}{1,2}\right\}
$$

It might appear that we could apply $\eta$ to it; however, as (11.1) shows, the result degenerates to a polyhedron with digonal holes.

There are no more polyhedra with square faces in the class $(2,3,2)$, and so our only further applications are to the class $(3,2,3)$. Thus we next have

$$
\left\{4, \frac{6}{1,3} \mid 3\right\} \stackrel{\eta}{\longmapsto}\left\{\frac{6}{1,3}, 6: \frac{6}{2,3}\right\},
$$

with the same group $[3,3,3] \rtimes C_{2}$.

Next, $\eta$ links up the two families of polyhedra derived from the extension $[3,4,3] \rtimes C_{2}$ of the group $[3,4,3]$. Here we have

$$
\{4,8 \mid 3\} \stackrel{\eta}{\longmapsto}\left\{\frac{8}{1,4}, 8: \frac{6}{2,3}\right\}, \quad\left\{4, \frac{8}{3} \mid 3\right\} \stackrel{\eta}{\longmapsto}\left\{\frac{8}{3,4}, \frac{8}{3}: \frac{6}{2,3}\right\},
$$

derived from the diagrams $\mathcal{D}_{2}\left(4,4, \frac{3}{2}\right)$ and $\mathcal{D}_{2}\left(\frac{4}{3}, \frac{4}{3}, \frac{3}{2}\right)$ in class $(2,3,2)$. Observe that these polyhedra all have the same vertices and group $[3,4,3] \rtimes C_{2}$; this follows from the existence of odd edge-circuits in the graphs of the first polyhedra in each pair. Naturally, the same then remains true when we further apply operations such as $\pi, \kappa$ and $\varphi_{3}$, which explains why we did not discuss the geometric structure of the polyhedra in the class $(2,3,2)$ in more detail in Section 8.

Last, consider a polyhedron with square faces derived by (7.2) from a 4-polytope (there are just three, coming from $\left\{3,3, q_{3}\right\}$ with $q_{3}=3,5$ or $\frac{5}{2}$ ). In such a case, $q$ is a fraction with an odd denominator, and the vertex-figure of the corresponding polyhedron of class $(2,3,2)$ will then collapse. A similar line of argument shows that the other possible polyhedra in class $(2,3,2)$ derived from diagrams with marks 5 or its fractions must also degenerate, because they can be obtained from such degenerate polyhedra in class $(3,2,3)$ by $\eta$.

We now come to the skewing operation $\sigma$; this applies to a regular polyhedron of Schläfli type $\{p, 4\}$. The result degenerates for toroidal polyhedra in the class $(2,3,2)$, 
and there are no others in that class to which it applies (in any event, since $\sigma=\pi \delta \eta \pi \delta$, and both $\pi$ and $\delta$ preserve this class, one is-in effect-reduced to considering $\eta$ ). It also degenerates for the few polyhedra in class $(2,2,2)$ to which it might be applicable; what goes wrong here is that the component regular projective polyhedra end up having different values of $q$.

However, $\sigma$ does work on class $(3,2,3)$; in fact, it yields polyhedra in class $(2,2,2)$. Moreover, as we shall see, it even actually applies to certain degenerate polyhedra in the class; recall that $\sigma$ really operates on groups. Now

$$
\left(R_{0}, R_{1}, R_{2}\right) \stackrel{\sigma}{\longmapsto}\left(R_{1}, R_{0} R_{2},\left(R_{1} R_{2}\right)^{2}\right)=:\left(S_{0}, S_{1}, S_{2}\right) .
$$

Thus, in terms of the diagram $\mathcal{D}_{1}(p, 2 ; r)$, the new generators $\left(S_{0}, S_{1}, S_{2}\right)$ are given by

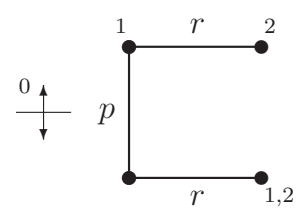

The labels on the diagram index the reflexions $S_{j}$; thus $S_{0}$ is the flip. Note that $S_{1}$ and $S_{2}$ are both products of two (commuting) hyperplane reflexions, one of which is shared between them; the new vertex-figure corresponds to the old hole. Further, $S_{0}=R_{1}$ is the flip in the original diagram; hence, subsequently applying $\kappa$ is equivalent to replacing the first mark $p$ in the diagram by $p^{\prime}$.

For the toroids, there should be no surprise to find that

$$
\mathcal{D}_{1}(2,2 ; r) \stackrel{\sigma}{\longmapsto}\left\{\frac{2 s}{t, s-t}, r ; \frac{2 s}{t, s-t}\right\}=\left\{2, r: r^{\prime \prime}\right\} \bowtie\left\{r^{\prime \prime}, r: 2\right\},
$$

with $r=s / t$.

Next, we have

$$
\mathcal{D}_{1}(3,2 ; 3) \stackrel{\sigma}{\longmapsto}\left\{\frac{10}{1,3}, 3 ; \frac{10}{1,3}\right\}=\left\{5,3: \frac{5}{2}\right\} \bowtie\left\{\frac{5}{2}, 3: 5\right\} .
$$

As we said in Section 10, the edge-graph of this polyhedron is the Petersen-type graph $G(10,3)$; it doubly covers the ordinary Petersen graph $G(5,2)$. Indeed, the latter is the graph of the hemi-dodecahedron, which is obtained as

$$
\mathcal{D}_{1}\left(\frac{3}{2}, 2 ; 3\right) \stackrel{\sigma}{\longmapsto}\left\{\frac{5}{1,2}, 3 ; \frac{5}{1,2}\right\}=\left\{5,3: \frac{5}{3}\right\} \bowtie\left\{\frac{5}{3}, 3: 5\right\} .
$$

This is an interesting example. First, it illustrates that a graph $\mathcal{D}_{1}(p, 2 ; r)$ giving a degenerate polyhedron in class $(3,2,3)$ can give rise to a non-degenerate one in class $(2,2,2)$. Second, note that the whole group is isomorphic to each of the component rotation groups in $\mathrm{SO}_{3}$.

Moving on, we have

$$
\left\{8, \frac{4}{1,2} \mid 3\right\} \stackrel{\sigma}{\longmapsto}\left\{\frac{24}{1,7}, 3: \frac{24}{1,7}\right\}=\{3,3: 4\} \bowtie\{4,3: 3\} .
$$

The geometry of this polyhedron is interesting and, incidentally, shows that it exists. It may be seen that its 192 vertices lie at the mid-points of the edges of the 24-cell 
$\{3,4,3\}$ and its reciprocal. A given vertex is joined to the mid-points of the edges of the corresponding triangular face of the reciprocal. Given two adjacent edges, there are two choices to continue to a third edge; these two choices correspond to the faces and the Petrie polygons (the polyhedron is self-Petrie).

In view of our earlier comment about $\kappa$, we have directly

$$
\left\{\frac{8}{3}, \frac{4}{1,2} \mid 3\right\} \stackrel{\sigma}{\longmapsto}\left\{\frac{24}{5,11}, 3: \frac{24}{5,11}\right\}=\left\{\frac{3}{2}, 3: 4\right\} \bowtie\left\{4,3: \frac{3}{2}\right\} .
$$

We have just recalled that the pentagonal diagrams, corresponding to regular polytopes such as $\left\{5, \frac{5}{2}, 5\right\}$, give rise to degenerate polyhedra in class $(3,2,3)$. What is surprising is that $\sigma$ does apply to these diagrams, yielding non-degenerate polyhedra in class $(2,2,2)$. We have already listed them in Section 10, but there are analogous geometric pictures to the previous example which describe their geometry, and thus establish their existence. So, for example,

$$
\left\{\frac{30}{1,11}, 5: \frac{30}{1,11}\right\}=\left\{\frac{5}{2}, 5: 3\right\} \bowtie\left\{3,5: \frac{5}{2}\right\}
$$

has its 720 vertices at the mid-points of the edges of the 600 -cell $\{3,3,5\}$ (or $\left\{5, \frac{5}{2}, 5\right\}$ ), and each vertex is joined by an edge to the mid-points of the edges of the pentagonal link of the original edge in the boundary complex of $\{3,3,5\}$.

\section{Acknowledgement}

Egon Schulte very carefully read through this paper before submission, and thereby enabled me to correct the usual trivial errors, as well as certain lacks of clarity in exposition. It is my pleasure to express my gratitude to him. Any mistakes which remain are, of course, my own.

\section{References}

1. J.L. Arocha, J. Bracho and L. Montejano, Regular projective polyhedra with planar faces, I. Aequationes Math. 59 (2000), 55-73.

2. J. Bracho, Regular projective polyhedra with planar faces, II. Aequationes Math. 59 (2000), 160-176.

3. H.S.M. Coxeter, Regular skew polyhedra in 3 and 4 dimensions and their topological analogues. Proc. London Math. Soc. (2) 43 (1937), 33-62. (Reprinted with amendments in Twelve Geometric Essays, Southern Illinois University Press, Carbondale, IL, 1968, pp. 76-105.)

4. H.S.M. Coxeter, Regular Polytopes (3rd edition). Dover, New York, 1973

5. H.S.M. Coxeter, Regular Complex Polytopes (2nd edition). Cambridge University Press, Cambridge, 1991.

6. H.S.M. Coxeter, Regular and semi-regular polytopes, III. Math. Z. 200 (1988), 3-45. (Reprinted in Kaleidoscopes: Selected Writings of H.S.M. Coxeter, eds. F.A. Sherk, P. McMullen, A.C. Thompson and A.I. Weiss, Wiley-Interscience, New York, 1995, pp. 313-355.)

7. H.S.M. Coxeter and W.O.J. Moser, Generators and Relations for Discrete Groups (4th edition). Springer, New York, 1980

8. A.W.M. Dress, A combinatorial theory of Grünbaum's new regular polyhedra, I: Grünbaum's new regular polyhedra and their automorphism group. Aequationes Math. 23 (1981), 252-265.

9. A.W.M. Dress, A combinatorial theory of Grünbaum's new regular polyhedra, II: complete enumeration. Aequationes Math. 29 (1985), 222-243.

10. P. Du Val, Homographies, Quaternions and Rotations. Oxford University Press, Oxford, 1964. 
11. L. Fejes Tóth, Reguläre Figuren. Akadémiai Kiadó, Budapest, 1965. (English translation: Regular Figures, Pergamon Press, Oxford, 1964.)

12. R. Frucht, J.E. Graver and M.E.Watkins, The groups of the generalized Petersen graphs. Math. Proc. Cambridge Philos. Soc. 70 (1971), 211-218.

13. B. Grünbaum, Regular polyhedra—old and new. Aequationes Math. 16 (1977), 1-20.

14. B. Grünbaum, Acoptic polyhedra. In Advances in Discrete and Computational Geometry, eds. B. Chazelle et al. Contemporary Mathematics, vol. 223. American Mathematical Society, Providence, RI, 1999, pp. 163-199.

15. P. McMullen, Realizations of regular polytopes. Aequationes Math. 37 (1989), 38-56.

16. P. McMullen, The regular polyhedra of type $\{p, 3\}$ with $2 p$ vertices. Geom. Dedicata 43 (1992), 285-289.

17. P. McMullen, The groups of the regular star-polytopes. Canad. J. Math. (2) 50 (1998), 426-448.

18. P. McMullen, Regular polytopes of full rank. Discrete Comput. Geom. 32 (2004), 1-35.

19. P. McMullen and B. R. Monson, Realizations of regular polytopes, II. Aequationes Math. 65 (2003), 102-112.

20. P. McMullen and E. Schulte, Constructions for regular polytopes. J. Combin. Theory Ser. A $\mathbf{5 3}$ (1990), $1-28$.

21. P. McMullen and E. Schulte, Regular polytopes in ordinary space. Discrete Comput. Geom. 17 (1997), 449-478.

22. P. McMullen and E. Schulte, Abstract Regular Polytopes. Encyclopedia of Mathematics and its Applications, vol. 92. Cambridge University Press, Cambridge, 2002.

23. P. McMullen and E. Schulte, Regular and chiral polytopes in low dimensions. The Coxeter LegacyReflections and Projections, eds. Chandler Davis and Erich W. Ellers. Fields Institute Communications, vol. 46. American Mathematical Society, Providence, RI, 2006, pp. 87-106.

24. B.R. Monson and A.I. Weiss, Realizations of regular toroidal maps. Canad. J. Math. (6) 51 (1999), $1240-1257$.

25. E. Schulte, Chiral polytopes in ordinary space, I. Discrete Comput. Geom. 32 (2004), 55-99.

26. E. Schulte, Chiral polytopes in ordinary space, II. Discrete Comput. Geom. 34 (2005), 181-229.

Received February 27, 2006, and in revised form June 2, 2006, and January 3, 2007.

Online publication July 13, 2007. 